\title{
Automatic Segmentation of Pelvic Cancers using Deep Learn- ing: State-of-the-Art Approaches and Challenges
}

\author{
Reza Kalantar', Gigin Lin ${ }^{2}$, Jessica M Winfield ${ }^{1,3}$, Christina Messiou ${ }^{1,3}$, Susan Lalondrelle ${ }^{1,4}$, Matthew D Black- \\ ledge ${ }^{1, *}$ and, Dow-Mu Koh ${ }^{1,3}$
}

1 Division of Radiotherapy and Imaging, The Institute of Cancer Research, London, United Kingdom; reza.kalantar@icr.ac.uk (R.K.); jessica.winfield@icr.ac.uk (J.M.W.); christina.messiou@icr.ac.uk (C.M.); susan.lalondrelle@rmh.nhs.uk (S.L.); matthew.blackledge@icr.ac.uk (M.D.B.); mu.koh@icr.ac.uk (D.M.K.)

2 Department of Medical Imaging and Intervention, Chang Gung Memorial Hospital at Linkou and Chang Gung University, 5 Fuhsing St., Guishan, Taoyuan, Taiwan; giginlin@cgmh.org.tw (G.L.)

3 Department of Radiology, The Royal Marsden Hospital, London, United Kingdom; jessica.winfield@rmh.nhs.uk (J.M.W.); christina.messiou@rmh.nhs.uk (C.M.); mu.koh@rmh.nhs.uk (D.M.K.)

4 Gynaecological Unit, The Royal Marsden Hospital, London, United Kingdom; susan.lalondrelle@rmh.nhs.uk (S.L.)

* Correspondence: matthew.blackledge@icr.ac.uk

\begin{abstract}
The recent rise of deep learning (DL) and its promising capabilities in capturing non-explicit detail from large datasets have attracted substantial research attention in the field of medical image processing. DL provides ground for technology development for computer-aided diagnosis and segmentation in radiology and radiation oncology. Amongst the anatomical locations where recent auto-segmentation algorithms have been employed, the pelvis remains one of the most challenging due to large intra- and inter-patient soft-tissue variabilities. This review provides a comprehensive and clinically-oriented overview of DL-based segmentation studies for bladder, prostate, cervical and rectal cancers, highlighting the key findings, challenges and limitations.
\end{abstract}

Keywords: Deep Learning; Pelvic Cancer Segmentation; Radiology; Radiation Oncology; Radiotherapy Planning

\section{Introduction}

Owning to the recent rise of high-resolution imaging modalities such as X-ray computed tomography (CT) and magnetic resonance imaging (MRI), medical practitioners rely on spatial visualization of internal organs to evaluate disease and make timely clinical decisions. Even though radiological assessment of imaging studies is still largely visual, based on domain knowledge and expertise, there is an increasing shift towards quantitative and volumetric disease assessment for precision medicine. This step requires accurate tissue segmentation, which can improve disease characterization through detection and division of abnormalities on images into semantically, biologically and/or clinically meaningful regions based on quantitative imaging measurements. Furthermore, the rapidly developing field of radiomics is also usually reliant on disease segmentation to obtain imaging signatures that can inform disease stratification or treatment outcomes. In radiation oncology, segmentation of the organs at risk (OARs) and target volumes are necessary steps to ensure optimal dose delivery to the tumor whilst avoiding toxicity to surrounding healthy tissues.

Traditionally, segmentation is performed manually by radiologists and radiation oncologists, which is time-consuming [1] and it may be associated with inter- and/or intraoperator variabilities [2,3]. In radiotherapy (RT), the time required for manual segmentation (MS) is also a rate-limiting step for adaptive radiotherapy (ART). ART is a treatment procedure that aims to account for temporal changes in patient anatomy and potentially tumor biology between each therapy fraction [4]. Furthermore, in RT clinics with limited resources and patient capacity, significant delays caused by MS were reported to adversely affect patient admissions as well as overall survival rates $[5,6]$. Therefore, 
significant research attention has been directed towards the development of fully-automatic segmentation algorithms to address these shortcomings and minimize burden on clinicians.

Recently, with remarkable advancements in hardware technology and the development of powerful graphical processing units (GPUs), deep learning (DL) techniques have emerged as potential revolutionary solutions for clinical applications due to their capabilities in learning intricate and complex features from very large medical datasets. Adoption of advanced DL techniques by clinics may lead to significant improvements in the current radiological and RT workflows. Computer-assisted segmentation technologies and their implementations are continuously evolving, providing the necessity for a comprehensive review of the state-of-the-art approaches developed for cancer diagnosis, treatment planning and response monitoring. Although previous publications have reviewed automatic medical image segmentation approaches [7-11] and some with particular focus in radiology [12] and radiation oncology [13,14], few studies have surveyed the clinical value and potential of DL-based segmentation approaches for different types of cancer in the pelvis. In this review, we provide an up-to-date overview of the current DL approaches undertaken for pelvic cancer segmentation, pinpoint key achievements and discuss limitations for potential adoption in the clinical practice.

\section{Background}

\subsection{What is Deep Learning?}

Artificial intelligence (AI) is the concept and theory of creating an ability for machines to learn and accomplish human-like intelligence [15]. DL is a sub-category of AI inspired by the human cognition system. Unlike traditional machine learning (ML) approaches that rely on pre-programmed set of instructions and manually-curated input data, DL offers the possibility of automatic feature extraction and learning from "raw data". Whilst many people perceive DL to be a 21st century invention, the first wave of research on how human/animal brains learn, also known as cybernetics, in fact started in the 1940s [16,17]. It was not until 1958 that the first fundamental component of artificial neural networks (ANNs), the perceptron, was developed and a single layer architecture was trained [18]. However, after a period of stagnation, the second wave of DL research, connectionism, began in the 1980s-1990s after the introduction of the backpropagation concept [19]. Backpropagation facilitated training of ANNs with one or two hidden layers for the first time. Nevertheless, due to lack of adequate computational processing power and increased pessimism on the real-world applications of DL in the mid-1990s, this wave of DL research was also short-lived. The current and third wave began in 2006 with development of convolutional neural networks (CNNs) [20] which allowed algorithms to be trained with significantly more efficiency than the traditional dense architectures (e.g. fully-connected networks). A key innovation in this approach was the realisation that sharing trained parameters (weights and biases of each perceptron) across the image through a convolution kernel enabled the development of much deeper networks for image processing than the previously available architectures [21]. Today, CNNs play a central role in AI design across a wide range of industries.

\subsection{Deep Learning in Oncology}

Whilst the interpretation of medical images is successfully undertaken by radiologists and radiation oncologists, their approach is often subjective and influenced by clinical experience. Depending on the prior experience, humans may not be able to fully account for the range of features present on scan images. This limitation can be exacerbated by the variable appearances of tumors in cancer patients. In recent times, AI has shown potential 
in automatic extraction of complex image features, not necessarily visible to the human eye [22]. After the introduction of CNNs, DL-based approaches were rapidly deployed for clinical research. In oncology, major applications of DL include tumor characterization (detection, segmentation and staging) [23-28], clinical outcome prediction [29,30], image synthesis [32,33] and RT dose-response modelling [34,35]. For an in-depth overview of AI applications beyond autosegmentation in radiology and radiation oncology, we refer the readers to previous excellent studies by Boldrini et al. [13] and Meyer et al. [14]. To illustrate the expansion of this field, an online search with keywords "deep learning" and "medical image segmentation" on Google Scholar for studies published between January 2016 to December 2020, revealed that the number of studies for DL-based segmentation research in medicine is rapidly rising. Additionally, the publication search with the additional keyword "cancer" indicated that cancer research has dictated a large proportion of recent DL-based medical image segmentation publications (Figure 1).

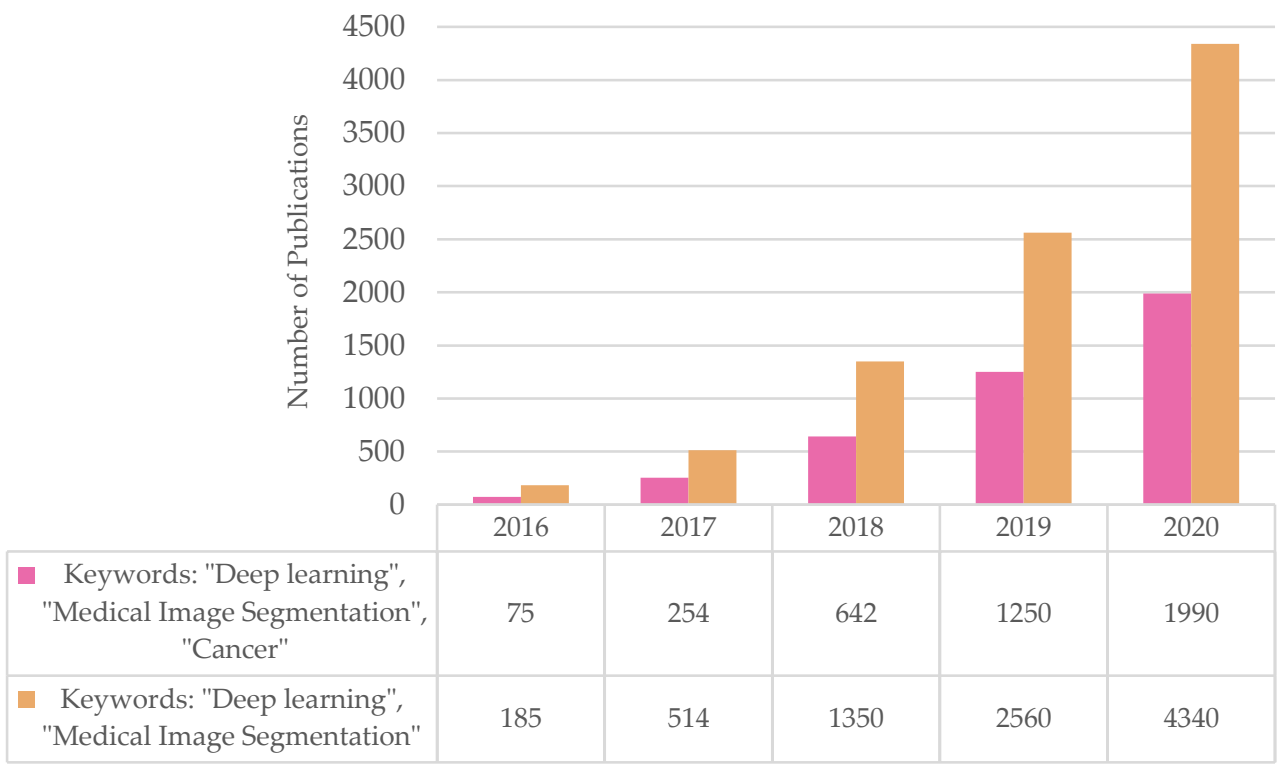

Figure 1. Rapid rise in the number of publications for DL-based segmentation research in medical imaging where almost half of studies were cancer-related between 2016 and 2020.

\subsection{Quantitative Imaging for Cancer Diagnosis, Characterization and Assessment of Treatment Response}

MRI is increasingly adopted by radiologists for diagnostic and therapeutic purposes [35-38]. MRI is especially advantageous for pelvic cancer diagnosis, as its higher contrastresolution compared with $\mathrm{CT}$ facilitates visualization and localization of suspicious lesions, delineation of disease extent, and subsequently enables targeted biopsy [39] and therapy planning [40]. Segmentation of target pelvic organs and tumors can be used to render disease volume, which can be further registered with patient scans from different imaging modalities for treatment planning. Tumor characterization is a broad term, which includes diagnosis, segmentation (differentiating from non-tumor tissues), staging (disease extent) and inferring its biological behavior. These applications may be enhanced by quantifying imaging characteristics such as size, shape and texture.

Tumor size measurement is important as it directs clinical decisions for the choice of treatment and evaluation of treatment response [41,42]. Disease monitoring is essential for assessing response to RT and chemotherapy treatments. The general workflow includes assessment of the tumor across longitudinal scans, and quantitative measurements according to predefined criteria (e.g. the Response Evaluation Criteria in Solid Tumors (RECIST), the World Health Organization (WHO) [43]. However, unidimensional tumor measurements can be limiting and volumetric assessment may be more robust. In 
addition, functional MRI techniques can be used to derive quantitative measurements that reflect on different aspects of tumor biology. For example, using diffusion-weighted (DW) MRI, the quantitative apparent diffusion coefficient (ADC) is an imaging biomarker related to tissue cellularity and has been shown to be promising for early evaluation of treatment response [44,45].

Radiomic analysis of tumors, a voxel-wise assessment using imaging features derived from CT or MR images, or quantitative MRI parametric maps (e.g. ADC), has shown promise for evaluating tumor aggressiveness [46] and for prognostic modelling [47]. Radiomics can be used to correlate phenotypical tumor characteristics to diagnostic and/or prognostic factors. However, applications as above are reliant on the accurate segmentation of tumors, which when undertaken manually, is both laborious and subjective [2,48]. Hence, automated and robust tumor segmentation tools are highly desirable for the rapid quantitative characterization of cancers.

\subsection{Radiotherapy Treatment (RT) Planning and Optimization}

$\mathrm{CT}$ remains the mainstay imaging modality for RT treatment planning due to its high acquisition speed, high spatial resolution and its ability to deliver relative electron density information. However, CT lacks the desired soft-tissue contrast for accurate delineation of organs and tumors where electron densities of neighboring structures are not significantly different. Therefore, in radiation oncology, gross tumor volumes (GTVs) are sometimes derived from MRI for more accurate delineations [49]. Within a treatment planning system (TPS), the radiation oncologist initially identifies the target volumes and OARs. A series of target volumes are defined according to the criteria reported by the International Commission on Radiation Units and Measurements (ICRU) [50], based on initial tumor identification expanded to include subclinical disease and finally a planning target volume (PTV) to account for day-to-day setup variation. Consistent identification of these target volumes during treatment using automated segmentation frameworks could help to reduce the expansion margins currently employed, and therefore limit irradiation of normal tissue. Despite defined delineation protocols, inter-observer variation in target delineation is the greatest source of uncertainty, necessitating an additional margin of error to be employed in creating the PTV [51]. Recently, image-guided radiation therapy (IGRT) techniques are increasingly attracting research attention to mitigate these shortcomings and allow clinicians to adapt treatment plans prior to and/or intra-fraction to objectively monitor the position of target volumes. In particular, ART is a potentially promising treatment procedure that suits tumor sites with large inter-fraction deformability (e.g. bladder, cervix, prostate, rectum); it allows better sparing of the OARs from radiation toxicity. However, the need for redefinition of regions of interest (ROIs) for each ART fraction poses a significant limitation in routine treatment workflows. Thus, fast, accurate and automatic segmentation of ROIs is considered the central requirement for ART for adoption in clinical practice.

\subsection{Automatic Image Segmentation}

Traditional segmentation algorithms were low-level image feature extractors (e.g. intensity-based and edge-based). Common methods included intensity thresholding, region growing and edge-detection that selected semantic image regions solely based on visual information from input images. Hence, more advanced mechanisms, such as uncertainty and optimization algorithms, were introduced to overcome the limitations associated with previous heuristic approaches. For instance, deformable models (e.g. active contours [52], level-set algorithms [53]) were developed to allow contours to expand/contract to include distinctive regions. The graph-based methods (e.g. graph cuts [54], watershed algorithm [55]) applied the principles of game theory for segmentations based on intervoxel relationships. Probability-based algorithms (e.g. Bayesian classifier [56,57], Gaussian mixture models, clustering, k-nearest neighbor [58], ANNs) were developed to 
automatically assign individual voxels to different classes. However, these approaches lacked contextual information which led to suboptimal segmentations. Although these algorithms can be combined with Markov-Random Field models to alleviate this drawback [59], the success of these techniques are strongly correlated with manual human interactions. Consequently, atlas-based approaches were proposed to incorporate prior knowledge in segmentation algorithms. Early atlas-based algorithms consisted of a single atlas (a manually defined set of regions on an existing reference image dataset) from which the contours from the reference image were transferred to the new image following deformable registration [60]. However, segmentation heavily relied on the registration accuracy and organ morphology, leading to suboptimal contours especially for patients with unusual anatomy.

Later approaches proposed the use of more advanced atlas selection techniques [61,62], selection of an atlas containing average patient anatomy information [63] and multi-atlas segmentation as prior knowledge $[60,64]$. Currently, multi-atlas algorithms are the most common techniques used in defining target tumor volumes [65]. Nonetheless, the major limitations with atlas-based methods remain the considerable computational and time constraints. In current oncological workflows, an array of software programs is available for automatic registration and segmentation of tumors using pre-defined templates and deformable contour propagations $[66,67]$. However, these programs are not suitable for pelvic cancers due to unclear boundaries between the gross tumor and subclinical malignant regions [68]; tumor contouring heavily relies on clinicians' experience.

Recently, DL-based segmentation methods have shown enormous potential in computer-assisted clinical applications due to their ability to learn complex information from very large datasets. Unlike traditional auto-segmentation approaches that rely on human defined heuristics, CNNs are able to automatically capture the pertinent information contained within existing (training) datasets needed for successful segmentation. CNNs are generally formed by stacking several layers (e.g. convolutional/deconvolutional, fullyconnected, pooling, upsampling layers), each of which perform a key operation on the input images (See Figure 2a for a basic CNN classification architecture). Conventionally, CNNs performed pixel/voxel-wise classifications to isolate independent pixels/voxels in order to form ROIs from images. However, this was computationally inefficient due to repetitive iterations of identical convolutional operations throughout images. In 2015, Long et al. [69] introduced fully-convolutional networks (FCNs) to mitigate the limitations with fully-connected layers (final set of layers in CNN) for extracting local spatial correlations. The FCN architecture includes symmetrical encoding and decoding paths which enable learning of both low- and high-level feature representations in images (Figure $2 b$ ). One of the most popular DL architectures used for medical image segmentation is UNet [70], which is a special type of an FCN with addition of skip connection pathways between encoders and decoders (Figure 2c). In recent years, many variations of UNet and FCNs have been published to enhance segmentation performance across a wide range of medical applications. Typical examples include 3D UNet [71], VNet [72], DeepMedic [73] and DeepLab [74]. Hereby, we direct the readers to [7], [9], [12] and [75] for comprehensive technical overview of DL architectures used in medical research. 


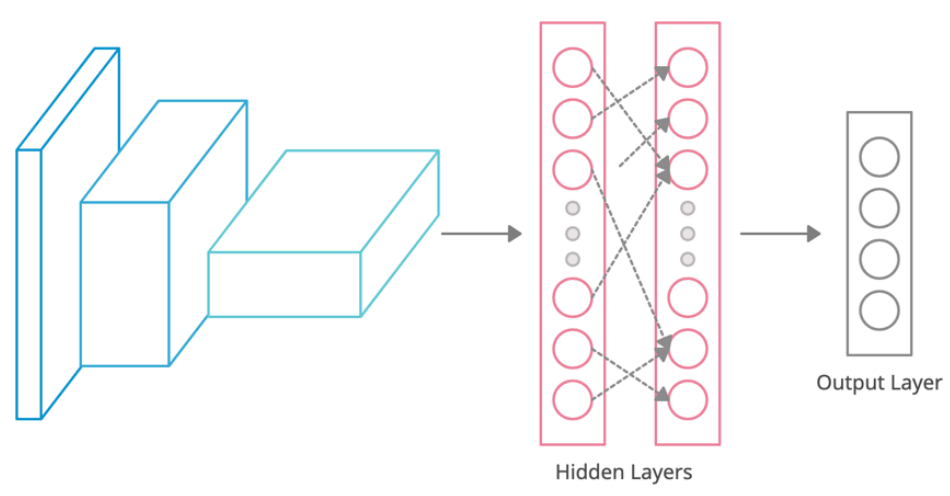

a. Convolutional Neural Network (CNN)

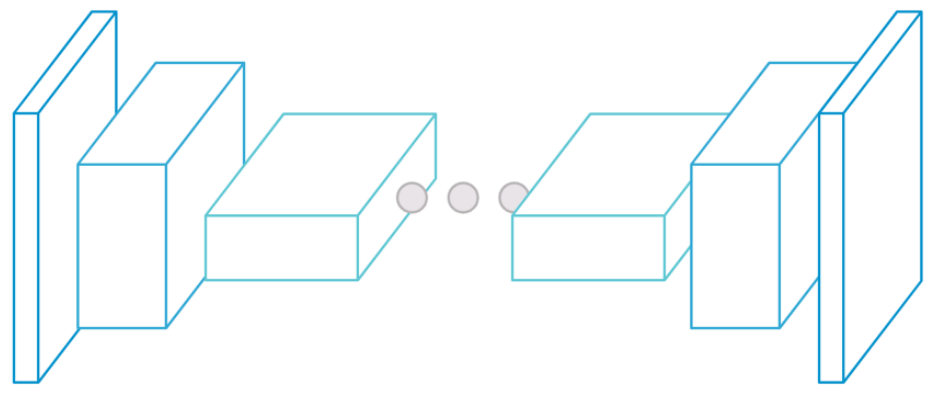

b. Fully-Convolutional Network (FCN)

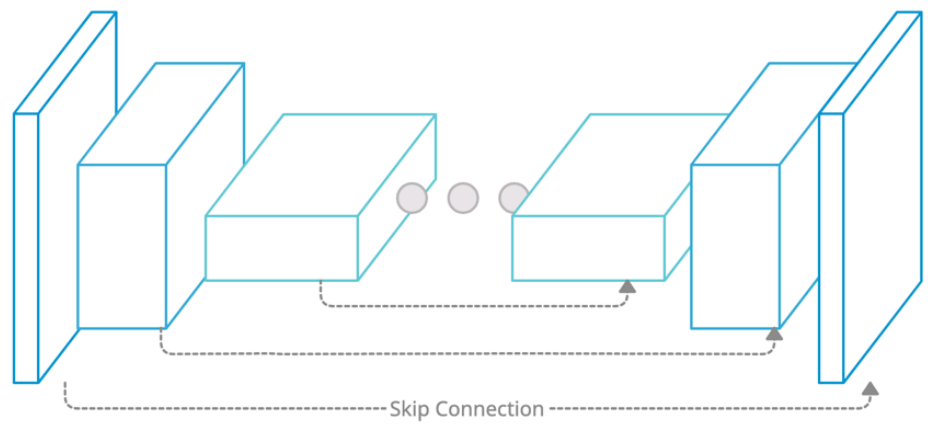

c. UNet

Figure 2. Illustration of (a) convolutional neural networks (CNN) with fully-connected final layers for classification tasks, (b) fully-convolutional network (FCN) for image-to-image or image-tomask translations, and (c) UNet architecture with skip connections between encoder and decoder in the network for more efficient feature extraction/reconstruction than FCN.

\subsubsection{Evaluating the Quality and Success of Segmentation}

One of the most broadly-used metrics for comparing automatically-generated contours with the ground-truth is the Dice similarity coefficient (DSC) [77]. DSC evaluates the overlap between two sets of contours (A and B) divided by their mean area. DSC ranges from 0 to 1 , where higher values correspond to more accurate segmentation results (Eq. 1). It considers both false positives and false negatives, therefore it is superior to accuracy which only incorporates correctly-identified pixels/voxels in images. Another variation of DSC reported in the literature is the surface Dice similarity coefficient (SDSC) [77] that, with addition of parameter $\tau$, incorporates inter-observer variabilities in measuring the overlap between two surfaces. Intersection-over-union (IoU) or Jaccard index (JI) is another segmentation metric reported in the literature [78] (Eq. 2). 


$$
\begin{gathered}
\mathrm{DSC}=\frac{2|\mathrm{~A} \cap \mathrm{B}|}{|\mathrm{A}|+|\mathrm{B}|} \\
\mathrm{IoU}=\frac{\mathrm{A} \cap \mathrm{B}}{\mathrm{A} \cup \mathrm{B}}
\end{gathered}
$$

One limitation associated with volume-based segmentation evaluation metrics (e.g. DSC, IoU) is the lack of sensitivity to the boundary of contours with potential spatial colocation. This is especially important in radiation oncology, where the contours of adjacent organs/target disease volumes may signify the difference between irradiated and at-risk regions. Therefore, distance-based metrics are used as additional indicators to assess segmented contours. The Hausdorff distance (HD) [79] is defined as follows (Eq. 3 and 4):

$$
\begin{gathered}
H D(A, B)=\max (h(A, B), h(B, A)) \\
h(A, B)=\max _{b \in B}\left(\min _{a \in A}\|a-b\|\right)
\end{gathered}
$$

where $h(A, B)$ is the largest distance from a point in A to the nearest point in $B$. HD is generally inversely correlated with segmentation accuracy. Additionally, the mean surface distance (MSD) is (Eq. 5):

$$
\operatorname{MSD}=\frac{1}{|A|+|B|}\left(\sum_{a \in A} \min _{b \in B} d(a, b)+\sum_{b \in B} \min _{a \in A} d(b, a)\right)
$$

where $d(a, b)$ corresponds to the distance between points $a$ and $b$.

In the following sections, we review DL-based segmentation publications for different cancer types within the pelvis.

\section{Methods}

The literature review in this study was conducted by initial article search from PubMed/Medline and ScienceDirect databases with keywords "deep learning", "segmentation", "cancer", "organs at risk", "radiation oncology", "radiology" and "radiotherapy", and subsequent manual reference check of the relevant publications. This approach aimed to create a clinically-oriented overview of the DL-based pelvic segmentation algorithms currently used in pelvic cancers. The exclusion criteria for the retrieved publications were as follows: no DL segmentation techniques used; segmentation applied to sites other than the pelvis; no training/validation of methods on real patient data; image modalities used other than CT and MRI; full-articles published in languages other than English; no clinical application focus or published outcome. Overall, we included 74 relevant studies on bladder, cervical, prostate and rectal cancer segmentation applications to present a comprehensive review of the state-of-the-art approaches (Table 1).

\section{Literature Review}

\subsection{Bladder Cancer}


Segmentation of the inner and outer bladder wall, and tumors on MRI plays an important role in diagnosis and staging of urinary bladder cancer as it provides excellent soft-tissue visualizations. On CT, bladder disease segmentation can provide clinicians with insight on cancer tumor progression and treatment response monitoring [80,81]. Bladder segmentation on MRI is a challenging task due to large inter-patient anatomical variations as well as imaging signal inhomogeneities in the urine caused by motion artefacts and unclear soft-tissue boundaries [82,83]. Additionally, segmentation difficulty amplifies with presence of cancer in the bladder. Previous studies performed automatic bladder segmentation using adaptive Markov-Random field [84], adaptive shape-prior constrained level-set [85] and statistical shape-based algorithms [86]. However, lack of generalizability due to large anatomical discrepancies in patient populations and the need for manual feature and parameter selection prevented their widespread clinical adoption.

To overcome this limitation, Ma et al. [81] developed a UNet-based framework that improved bladder segmentation on CT compared with their previous combined CNN and level-set segmentation algorithm [86], particularly in lower resolution images and scans from patients with locally-advanced urinary bladder cancer. However, the authors reported that contrast-enhanced CT images added more complexity to segmentation due to variable appearance of the bladder based on the effects of urine motion effect and filling from excreted contrast material. Xu et al. [87] proposed a 3D bladder segmentation framework on CT involving a fully-connected conditional random fields recurrent neural network (CRF-CNN) and fine-localized bladder probability maps; they reported that their approach outperformed the state-of-the-art VNet algorithm for volumetric segmentation of the bladder. On the other hand, only the study published by Dolz et al. [88] incorporated DL for bladder cancer segmentation on MRI. The authors developed a UNet-based 2D framework to perform multi-region semantic bladder segmentation and reported that this approach outperformed traditional non-DL autosegmentation techniques. We hypothesize that the paucity of published studies for use of DL in bladder cancer segmentation may be due to the lack of public and annotated datasets, as well as the lower prevalence of the disease compared with other pelvic cancers (see Table 1 and Figure 3).

Table 1. Summary of previous publications using DL-based automatic segmentation separated by pelvic anatomical regions (Bladder: 6, Cervix: 7, Prostate: 52, Rectum: 9 studies). The DSC and IoU are shown, where reported, with the DSC metrics in bold (for studies with multiple test results, the metrics calculated on public/external databases are presented). For studies that reported neither DSC nor IoU, the metrics used by the authors are included. MRI acquisition modes (2D, 3D) were retrieved based on the information provided in each published article and/or supplementary documents.

\begin{tabular}{|c|c|c|c|c|c|c|}
\hline $\begin{array}{c}\text { Image } \\
\text { Modality } \\
\text { (MR } \\
\text { Acquisition } \\
\text { Mode) }\end{array}$ & $\begin{array}{c}\text { Deep Learning } \\
\text { Strategy }\end{array}$ & $\begin{array}{c}\text { DL } \\
\text { Network } \\
\text { Dimension }\end{array}$ & $\begin{array}{c}\text { Number of } \\
\text { Patients } \\
\text { (Train/Test) }\end{array}$ & Segmentation Evaluation Metrics & Year & Reference \\
\hline \multicolumn{7}{|c|}{ Bladder Cancer } \\
\hline $\mathrm{CT}$ & UNet & $2 \mathrm{D} / 3 \mathrm{D}$ & $81 / 92$ & Bladder (IoU: 0.85/0.82) & 2019 & [81] \\
\hline $\mathrm{CT}$ & $\begin{array}{c}\mathrm{CNN}+\mathrm{FCN}(\mathrm{CRF}- \\
\mathrm{RNN})\end{array}$ & $3 \mathrm{D}$ & $100 / 24$ & Bladder (DSC: 0.92) & 2018 & [87] \\
\hline CT & $\mathrm{CNN}$ & $2 \mathrm{D}$ & $\begin{array}{c}62 \text { leave- } \\
\text { one-out } \mathrm{CV}\end{array}$ & Bladder Tumor (AUC: 0.73) & 2016 & [80] \\
\hline $\mathrm{CT}$ & $\mathrm{CNN}$ & $2 \mathrm{D}$ & $81 / 92$ & Bladder (IoU: 0.76) & 2016 & [86] \\
\hline
\end{tabular}




\begin{tabular}{c|c|c|c|c|c|c}
\hline $\mathrm{T}_{2} \mathrm{~W}$ (2D), & $\begin{array}{c}\text { AE + modified } \\
\text { residual network } \\
\text { DW (2D) MRI }\end{array}$ & 2D & $144 / 25$ & Bladder Wall (DSC: 0.85) & 2020 & [89] \\
\hline & $\begin{array}{c}\text { UNet with } \\
\text { T2W MRI (3D) }\end{array}$ & $\begin{array}{c}\text { progressive dilated } \\
\text { convolutions (UNet- } \\
\text { Progressive) }\end{array}$ & 2D & $40 / 15$ & Outer Wall (DSC: 0.83), Inner Wall (DSC: 0.98) & 2018 \\
\hline
\end{tabular}

\section{Cervical Cancer}

\begin{tabular}{|c|c|c|c|c|c|c|}
\hline $\mathrm{CT}$ & $\begin{array}{c}\text { UNet with context } \\
\text { aggregation blocks } \\
\text { (CabUNet) }\end{array}$ & $2 \mathrm{D}$ & $77 / 14$ & $\begin{array}{l}\text { Bladder (DSC: 0.90), Bone Marrow (DSC: 0.85), } \\
\text { L Fem. Head (DSC: 0.90), R Fem. Head (DSC: } \\
\text { 0.90), Rectum (DSC: 0.79), Small Intestine (DSC: } \\
\text { 0.83), Spinal Cord (DSC: 0.82) }\end{array}$ & 2020 & {$[90]$} \\
\hline $\mathrm{CT}$ & $\begin{array}{c}\text { Dual path UNet } \\
\text { (DpnUNet) }\end{array}$ & $2.5 \mathrm{D}$ & $\begin{array}{c}210 \text { five-fold } \\
\text { CV }\end{array}$ & $\begin{array}{l}\text { CTV (DSC: 0.86), Bladder (DSC: 0.91), } \\
\text { Bone Marrow (DSC: 0.85), L Fem. Head (DSC: } \\
\text { 0.90), R Fem. Head (DSC: 0.90), Rectum (DSC: } \\
\text { 0.82), Bowel Bag (DSC: } 0.85) \text {, Spinal Cord (DSC: } \\
0.82 \text { ) }\end{array}$ & 2020 & {$[91]$} \\
\hline $\mathrm{CT}$ & UNet & $3 \mathrm{D}$ & $100 / 25$ & $\begin{array}{l}\text { CTV (DSC: 0.86), Bladder (DSC: 0.88), } \\
\text { Rectum (DSC: 0.81), L Fem. Head (DSC: 0.88), } \\
\text { R Fem. Head (DSC: 0.88), Small Intestine (DSC: } \\
\text { 0.86) }\end{array}$ & 2020 & {$[92]$} \\
\hline $\mathrm{CT}$ & $\begin{array}{l}\text { UNet with residual } \\
\text { connection, dilated } \\
\text { convolution and } \\
\text { deep supervision } \\
\text { (DSD-UNet) }\end{array}$ & $3 \mathrm{D}$ & $73 / 18$ & $\begin{array}{l}\text { High-risk CTV (DSC: 0.82, IOU: 0.72), } \\
\text { Bladder (DSC: 0.86, IOU: 0.77), } \\
\text { Rectum (DSC: } \mathbf{0 . 8 2} \text {, IOU: 0.71), } \\
\text { Small Intestine (DSC: } \mathbf{0 . 8 0} \text {, IOU: 0.69), } \\
\text { Sigmoid (DSC: } \mathbf{0 . 6 4} \text {, IOU: 0.52) }\end{array}$ & 2020 & [93] \\
\hline $\mathrm{CT}$ & VNet & $3 \mathrm{D}$ & $\begin{array}{c}2464 / 140 \\
(+30 \text { external } \\
\text { test patients) }\end{array}$ & $\begin{array}{l}\text { Primary CTV (UteroCervix) (DSC: 0.85), } \\
\text { Nodal CTV (DSC: 0.86), PAN CTV (DSC: 0.76), } \\
\text { Bladder (DSC: 0.89), Rectum (DSC: 0.81), } \\
\text { Spinal Cord (DSC: 0.90), L Femur (DSC: 0.94), } \\
\text { R Femur (DSC: 0.93), L Kidney (DSC: 0.94), } \\
\text { R Kidney (DSC: 0.95), Pelvic Bone (DSC: 0.93), } \\
\text { Sacrum (DSC: 0.91), L4 Vertebral Body (DSC: } \\
\text { 0.91), } \\
\text { L5 Vertebral Body (DSC: 0.90) }\end{array}$ & 2020 & {$[94]$} \\
\hline $\begin{array}{c}\text { MRI } \\
\text { (unspecified) }\end{array}$ & Mask R-CNN & $2 \mathrm{D}$ & $\begin{array}{l}5 \text { (646 } \\
\text { images split } \\
\text { 9:1 for } \\
\text { training and } \\
\text { testing) }\end{array}$ & $\begin{array}{c}\text { GTV + Cervix (DSC: 0.84), Uterus (DSC: 0.92), } \\
\text { Sigmoid (DSC: 0.89), Bladder (DSC: 0.90), } \\
\text { Rectum (DSC: 0.89), Parametrium (DSC: 0.66), } \\
\text { Vagina (DSC: 0.71), Mesorectum (DSC: 0.68), } \\
\text { Femur (DSC: 0.81) }\end{array}$ & 2019 & {$[95]$} \\
\hline DW MRI (2D) & UNet & $2 \mathrm{D}$ & $144 / 25$ & Cervical Tumor (DSC: 0.82 ) & 2019 & [96] \\
\hline
\end{tabular}




\begin{tabular}{|c|c|c|c|c|c|c|}
\hline \multicolumn{7}{|c|}{ Prostate Cancer } \\
\hline $\mathrm{CT}$ & $\begin{array}{l}\text { UNet (External } \\
\text { commercial } \\
\text { software) }\end{array}$ & $2 \mathrm{D}$ & $328 / 20$ & $\begin{array}{l}\text { Prostate (DSC: 0.79), Bladder (DSC: 0.97), } \\
\text { Rectum (DSC: 0.78), Fem. Head (DSC: 0.91), } \\
\text { Seminal Vesicles (DSC: 0.64) }\end{array}$ & 2020 & [97] \\
\hline CT & UNet & $3 \mathrm{D}$ & $900 / 30$ & $\begin{array}{l}\text { Prostate (DSC: 0.82), Bladder (DSC: 0.93), } \\
\text { Rectum (DSC: 0.84), L Fem. Head (DSC: 0.68), } \\
\text { R Fem. Head (DSC: 0.69), } \\
\text { Lymph Nodes (DSC: } 0.80), \\
\text { Seminal Vesicles (DSC: } 0.72)\end{array}$ & 2020 & [98] \\
\hline CT & $\begin{array}{l}\text { High-resolution } \\
\text { multi-scale encoder- } \\
\text { decoder network } \\
\text { (HMEDN) }\end{array}$ & $2 \mathrm{D}$ & $180 / 100$ & $\begin{array}{l}\text { Prostate (DSC: 0.88), Bladder (DSC: 0.94), } \\
\text { Rectum (DSC: 0.87) }\end{array}$ & 2019 & [99] \\
\hline $\begin{array}{l}\text { CT/ } \\
\text { Synthetic } \mathrm{T}_{2} \mathrm{~W} \\
\text { MRI }\end{array}$ & $\begin{array}{l}\text { CT-to-MR synthesis } \\
\text { + Deep Attention } \\
\text { UNet (DAUNet) }\end{array}$ & $3 \mathrm{D}$ & $\begin{array}{l}\text { 112/28 five- } \\
\text { fold CV }\end{array}$ & $\begin{array}{c}\text { Prostate (DSC: 0.87), Bladder (DSC: 0.95), } \\
\text { Rectum (DSC: 0.89) }\end{array}$ & 2019 & [100] \\
\hline $\mathrm{CT}$ & Modified UNet & $3 \mathrm{D}$ & $\begin{array}{l}313 \text { five-fold } \\
\text { CV }\end{array}$ & $\begin{array}{l}\text { Prostate: (DSC: 0.89), Bladder: (DSC: 0.94), } \\
\text { Rectum: (DSC: 0.89) }\end{array}$ & 2019 & [101] \\
\hline $\mathrm{CT}$ & $\begin{array}{c}\text { Deep Neural } \\
\text { Network (DNN) }\end{array}$ & $3 \mathrm{D}$ & $771 / 140$ & Prostate (DSC: $\mathbf{0 . 8 8}$ ) & 2019 & [102] \\
\hline $\mathrm{CT}$ & $\begin{array}{c}\text { Deeply-supervised } \\
\text { attention-enabled } \\
\text { boosted } \\
\text { convolutional neural } \\
\text { network } \\
\text { (DAB-CNN) }\end{array}$ & $3 \mathrm{D}$ & $80 / 20$ & $\begin{array}{l}\text { Prostate (DSC: 0.90), Bladder (DSC: 0.93), } \\
\text { Rectum (DSC: 0.83), Penile bulb (DSC: 0.72) }\end{array}$ & 2019 & [103] \\
\hline $\mathrm{CT}$ & $\begin{array}{l}\text { Distinctive curve } \\
\text { guided fully } \\
\text { convolutional } \\
\text { network (FCN) }\end{array}$ & $2 \mathrm{D}$ & $\begin{array}{l}313 \text { five-fold } \\
\text { CV }\end{array}$ & $\begin{array}{c}\text { Prostate (DSC: 0.89), Bladder (DSC: 0.94), } \\
\text { Rectum (DSC: 0.89) }\end{array}$ & 2019 & [104] \\
\hline $\mathrm{CT}$ & UNet & $2 \mathrm{D}$ & $60 / 25$ & $\begin{array}{c}\text { Prostate: (DSC: } 0.88) \text {, Bladder: DSC: 0.95), } \\
\text { Rectum: (DSC: 0.92) }\end{array}$ & 2018 & [105] \\
\hline $\mathrm{CT}$ & $\begin{array}{l}\text { 2D UNet + 3D UNet } \\
\text { with aggregated } \\
\text { residual networks } \\
\quad(\text { ResNeXt) }\end{array}$ & $2 \mathrm{D} / 3 \mathrm{D}$ & $\begin{array}{l}\text { 108/28 four- } \\
\text { fold CV }\end{array}$ & $\begin{array}{c}\text { Prostate (DSC: 0.90), Bladder (DSC: 0.95), } \\
\text { Rectum (DSC: 0.84), L Fem. Head (DSC: 0.96), } \\
\text { R Fem. Head (DSC: 0.95) }\end{array}$ & 2018 & [106] \\
\hline $\mathrm{CT}$ & $\begin{array}{l}\mathrm{CNN}+\text { multi-atlas } \\
\text { fusion }\end{array}$ & $2 \mathrm{D}$ & $\begin{array}{l}92 \text { five-fold } \\
\qquad \mathrm{CV}\end{array}$ & Prostate (DSC: 0.86 ) & 2017 & [26] \\
\hline
\end{tabular}




\begin{tabular}{|c|c|c|c|c|c|c|}
\hline $\mathrm{CT}$ & $\begin{array}{l}\text { FCN (based on } \\
\text { LeNet) }\end{array}$ & $2 \mathrm{D}$ & $\begin{array}{l}22 \text { two-fold } \\
\mathrm{CV}\end{array}$ & Prostate (DSC: 0.89) & 2017 & [107] \\
\hline $\mathrm{T}_{2} \mathrm{~W}$ MRI (2D) & $\begin{array}{c}\text { Adversarial } \\
\text { pyramid anisotropic } \\
\text { convolutional deep } \\
\text { neural network } \\
\text { (APA-Net) }\end{array}$ & $3 \mathrm{D}$ & $\begin{array}{l}110 \text { three- } \\
\text { fold CV }\end{array}$ & Whole Prostate Gland (DSC: 0.90) & 2020 & [108] \\
\hline $\begin{array}{l}\mathrm{T}_{2} \mathrm{~W} \text { MRI } \\
(2 \mathrm{D} / 3 \mathrm{D})\end{array}$ & DeeplabV3+ & $2 \mathrm{D}$ & 40 & $\begin{array}{l}\text { Prostate Central Gland (DSC: 0.81), } \\
\text { Peripheral Zone (DSC: } 0.70)\end{array}$ & 2020 & [109] \\
\hline $\begin{array}{c}\mathrm{T}_{2} \mathrm{~W}(2 \mathrm{D}) \\
\mathrm{DW}(2 \mathrm{D}) \mathrm{MRI}\end{array}$ & $\begin{array}{c}\text { Conditional GAN } \\
\text { (cGAN)/Cycle- } \\
\text { consistent GAN } \\
\text { (Cycle-GAN) }\end{array}$ & $2 \mathrm{D}$ & $40 / 50$ & Whole Prostate Gland (DSC: 0.75) & 2020 & [110] \\
\hline $\begin{array}{c}\mathrm{T}_{2} \mathrm{~W}(2 \mathrm{D}) \\
\mathrm{DW}(2 \mathrm{D}) \mathrm{MRI}\end{array}$ & Mask R-CNN & $2 \mathrm{D}$ & $\begin{array}{c}54 / 16(+12 \\
\text { external test } \\
\text { patients })\end{array}$ & $\begin{array}{c}\text { Whole Prostate Gland (DSC: 0.86), } \\
\text { Prostate Tumor (DSC: 0.56) }\end{array}$ & 2020 & [111] \\
\hline $\mathrm{T}_{2} \mathrm{~W}$ MRI (2D) & $\begin{array}{c}\text { Boundary-weighted } \\
\text { domain adaptive } \\
\text { neural network } \\
\text { (BOWDA-Net) }\end{array}$ & $3 \mathrm{D}$ & $40 / 146$ & $\begin{array}{c}\text { Whole Prostate Gland (DSC: 0.91) } \\
\text { Prostate Base (DSC: 0.89) } \\
\text { Prostate Apex (DSC: 0.89) }\end{array}$ & 2020 & [112] \\
\hline $\mathrm{T}_{2} \mathrm{~W}$ MRI (2D) & $\begin{array}{c}\text { Graph convolutional } \\
\text { network } \\
(\mathrm{GCN})\end{array}$ & $2 \mathrm{D}$ & $\begin{array}{l}140 \text { five-fold } \\
\qquad \mathrm{CV}\end{array}$ & Whole Prostate Gland (DSC: $\mathbf{0 . 9 3 )}$ & 2020 & [113] \\
\hline $\mathrm{T}_{2} \mathrm{~W}$ MRI (2D) & Dense UNet & $2 \mathrm{D}$ & $\begin{array}{l}\text { 141/47 } \\
\text { four-fold CV }\end{array}$ & $\begin{array}{c}\text { Whole Prostate Gland (DSC: 0.92), } \\
\text { Central Gland (DSC: 0.89), } \\
\text { Peripheral Zone (DSC: 0.78) }\end{array}$ & 2020 & [114] \\
\hline $\mathrm{T}_{2} \mathrm{~W}$ MRI (2D) & UNet/Pix2pix & $2 \mathrm{D}$ & $\begin{array}{l}40 \text { four-fold } \\
\qquad \mathrm{CV}\end{array}$ & $\begin{array}{c}\text { Prostate Central Gland (DSC: 0.86-0.88), } \\
\text { Peripheral Zone (DSC: } \mathbf{0 . 9 0 - 0 . 8 3 )}\end{array}$ & 2020 & [115] \\
\hline $\begin{array}{l}\mathrm{T}_{1} \mathrm{~W}(3 \mathrm{D}), \mathrm{T}_{2} \mathrm{~W} \\
\text { (unspecified) } \\
\text { MRI }\end{array}$ & $\begin{array}{l}\text { Multi-scale } \\
\text { DeepMedic }\end{array}$ & 3D & $\begin{array}{l}\text { 97/53 three- } \\
\text { fold CV }\end{array}$ & $\begin{array}{l}\text { Bladder (DSC: 0.96), Rectum (DSC: 0.88), } \\
\text { L femur (DSC: 0.97), R femur (DSC: 0.97) }\end{array}$ & 2020 & [116] \\
\hline $\mathrm{T}_{2} \mathrm{~W}$ MRI (2D) & $\begin{array}{l}\text { Cascaded dual } \\
\text { attention network } \\
\text { (CDA-Net) }\end{array}$ & $3 \mathrm{D}$ & $40 / 109$ & Whole Prostate Gland (DSC: 0.92) & 2020 & [117] \\
\hline $\mathrm{T}_{2} \mathrm{~W}$ MRI (2D) & $\begin{array}{l}\text { Encoder-Decoder } \\
\text { structure with dense } \\
\text { dilated spatial } \\
\text { pyramid pooling } \\
\text { (DDSPP) }\end{array}$ & $2 \mathrm{D}$ & 150 & Whole Prostate Gland (DSC: 0.95) & 2019 & [118] \\
\hline
\end{tabular}




\begin{tabular}{|c|c|c|c|c|c|c|}
\hline $\begin{array}{c}\mathrm{T}_{2} \mathrm{~W}(2 \mathrm{D}) \\
\mathrm{DW}(2 \mathrm{D}) \mathrm{MRI}\end{array}$ & Mask R-CNN & $2 \mathrm{D}$ & $\begin{array}{l}36 \text { (split } \\
\text { 7:2:1 for } \\
\text { training, } \\
\text { validation } \\
\text { and testing) }\end{array}$ & $\begin{array}{c}\text { Whole Prostate Gland (IoU: 0.84), } \\
\text { Prostate Tumor (IoU: 0.40), } \\
\text { Central Gland (IoU: 0.78), } \\
\text { Peripheral Zone (IoU: 0.51) }\end{array}$ & 2019 & [119] \\
\hline $\begin{array}{c}\mathrm{T}_{2} \mathrm{~W}(2 \mathrm{D}) \\
\mathrm{DW}(2 \mathrm{D}) \mathrm{MRI}\end{array}$ & UNet & $2 \mathrm{D}$ & $100 / 125$ & $\begin{array}{l}\text { Whole Prostate Gland (DSC: 0.84), } \\
\text { Central Gland (DSC: 0.78), } \\
\text { Peripheral Zone (DSC: 0.69) }\end{array}$ & 2019 & {$[120]$} \\
\hline $\mathrm{T}_{2} \mathrm{~W}$ MRI (2D) & $\begin{array}{l}\text { FCN with feature } \\
\text { pyramid attention }\end{array}$ & $2 \mathrm{D}$ & $\begin{array}{c}250 / 63(+46 \\
\text { external test } \\
\text { patients) }\end{array}$ & $\begin{array}{c}\text { Prostate Transition Zone (DSC: 0.79), } \\
\text { Peripheral zone (DSC: 0.74) }\end{array}$ & 2019 & [121] \\
\hline $\mathrm{T}_{2} \mathrm{~W}$ MRI (3D) & $\begin{array}{l}\text { Spatially-varying } \\
\text { stochastic residual } \\
\text { adversarial network } \\
\text { (STRAINet) }\end{array}$ & $3 \mathrm{D}$ & $\begin{array}{l}50 \text { five-fold } \\
\qquad \mathrm{CV}\end{array}$ & $\begin{array}{l}\text { Whole Prostate Gland (DSC: 0.91), } \\
\text { Bladder (DSC: 0.97), Rectum (DSC: 0.91) }\end{array}$ & 2019 & [122] \\
\hline $\mathrm{T}_{2} \mathrm{~W}$ MRI (2D) & $\begin{array}{l}\text { UNet with } \\
\text { "combo loss" }\end{array}$ & $3 \mathrm{D}$ & $700 / 258$ & Whole Prostate Gland (DSC: 0.91) & 2019 & [123] \\
\hline $\begin{array}{c}\mathrm{T}_{2} \mathrm{~W} \text { MRI } \\
\text { (unspecified) }\end{array}$ & DeepLabV3+ & $2 \mathrm{D}$ & $40 / 50$ & $\begin{array}{l}\text { CTV (DSC: 0.83), Bladder (DSC: 0.93), } \\
\text { Rectum (DSC: 0.82), Penile Bulb (DSC: 0.74), } \\
\text { Urethra (DSC: 0.69), Rectal Spacer (DSC: 0.81) }\end{array}$ & 2019 & [124] \\
\hline $\mathrm{T}_{2} \mathrm{~W}$ MRI (2D) & $\begin{array}{l}\text { VNet + variational } \\
\text { methods }\end{array}$ & $3 \mathrm{D}$ & 85 & Whole Prostate Gland (DSC: 0.64) & 2019 & [125] \\
\hline $\mathrm{T}_{2} \mathrm{~W}$ MRI (2D) & $\begin{array}{l}\text { Propagation Deep } \\
\text { Neural Network } \\
\text { (P-DNN) }\end{array}$ & $2 \mathrm{D}$ & $50 / 30$ & Whole Prostate Gland: (DSC: 0.84) & 2019 & [126] \\
\hline $\begin{array}{c}\mathrm{T}_{2} \mathrm{~W}(2 \mathrm{D}) \\
\mathrm{DW}(2 \mathrm{D}) \mathrm{MRI}\end{array}$ & Cascaded UNet & $2 \mathrm{D}$ & $76 / 51$ & $\begin{array}{c}\text { Whole Prostate Gland (DSC: 0.92), } \\
\text { Peripheral zone (DSC: 0.79) }\end{array}$ & 2019 & [127] \\
\hline $\mathrm{T}_{2} \mathrm{~W}$ MRI (3D) & Multi-view CNN & $2 \mathrm{D}$ & $\begin{array}{l}19 \text { leave- } \\
\text { one-out }\end{array}$ & $\begin{array}{c}\text { Prostate Tumor (DSC: 0.92, IoU: 0.67), } \\
\text { Prostate Central Gland (IoU: 0.65), } \\
\text { Peripheral Zone (IoU: 0.59) }\end{array}$ & 2019 & [128] \\
\hline $\mathrm{T}_{2} \mathrm{~W}$ MRI (2D) & $\begin{array}{c}\text { Investigative CNN } \\
\text { study (UNet, VNet, } \\
\text { HighRes3dNet, } \\
\text { HolisticNet, Dense } \\
\text { VNet, Adapted } \\
\text { UNet) }\end{array}$ & $3 \mathrm{D}$ & $173 / 59$ & Whole Prostate Gland (DSC: 0.87 ) & 2019 & [129] \\
\hline $\mathrm{T}_{2} \mathrm{~W}$ MRI (2D) & ZNet & $2 \mathrm{D}$ & $45 / 30$ & Whole Prostate Gland (DSC: 0.90 ) & 2019 & [130] \\
\hline
\end{tabular}




\begin{tabular}{|c|c|c|c|c|c|c|}
\hline $\mathrm{T}_{2} \mathrm{~W}$ MRI (3D) & FCN & $3 \mathrm{D}$ & $60 / 10$ & $\begin{array}{l}\text { Whole Prostate Gland (DSC: 0.89), } \\
\text { Bladder (DSC: 0.95), Rectum (DSC: 0.88) }\end{array}$ & 2018 & [131] \\
\hline $\mathrm{T}_{2} \mathrm{~W}$ MRI (2D) & SegNet & $2 \mathrm{D}$ & $\begin{array}{c}16 / 5(+19 \\
\text { external test } \\
\text { patients })\end{array}$ & Whole Prostate Gland (DSC: 0.75) & 2018 & [132] \\
\hline $\mathrm{T}_{2} \mathrm{~W}$ MRI (2D) & $\begin{array}{l}\text { CNN + Boundary } \\
\text { Detection }\end{array}$ & $3 \mathrm{D}$ & $\begin{array}{l}50 \text { five-fold } \\
\qquad \mathrm{CV}\end{array}$ & Whole Prostate Gland (DSC: $\mathbf{0 . 9 0}$ ) & 2018 & [133] \\
\hline DCE MRI (3D) & UNet + LSTM & $3 \mathrm{D}$ & $\begin{array}{l}(15 / 2) \text { three- } \\
\text { fold CV }\end{array}$ & Whole Prostate Gland (DSC: $\mathbf{0 . 8 6}$ ) & 2018 & [134] \\
\hline $\mathrm{T}_{2} \mathrm{~W}$ MRI (2D) & FCN & $2 \mathrm{D}$ & $50 / 30$ & Whole Prostate Gland (DSC: 0.87 ) & 2018 & [135] \\
\hline $\mathrm{T}_{2} \mathrm{~W}$ MRI (2D) & $\mathrm{CNN}$ & $2 \mathrm{D}$ & 20 & Whole Prostate Gland (DSC: 0.85) & 2018 & [25] \\
\hline $\mathrm{T}_{2} \mathrm{~W}$ MRI (2D) & CNN (PSNet) & $3 \mathrm{D}$ & $\begin{array}{l}\text { 112/28 five- } \\
\text { fold CV }\end{array}$ & Whole Prostate Gland (DSC: 0.85) & 2018 & {$[24]$} \\
\hline $\begin{array}{c}\mathrm{T}_{2} \mathrm{~W}(2 \mathrm{D}) \\
\mathrm{DW}(2 \mathrm{D}) \mathrm{MRI}\end{array}$ & $\begin{array}{c}\text { Deep dense } \\
\text { multi-path CNN }\end{array}$ & $3 \mathrm{D}$ & $\begin{array}{c}100 / 50(+30 \\
\text { external test } \\
\text { patients })\end{array}$ & Whole Prostate Gland (DSC: 0.95) & 2018 & [136] \\
\hline $\mathrm{T}_{2} \mathrm{~W}$ MRI (2D) & UNet & $3 \mathrm{D}$ & 26 & Whole Prostate Gland (DSC: 0.88) & 2018 & [137] \\
\hline $\mathrm{T}_{2} \mathrm{~W}$ MRI (2D) & $\begin{array}{l}\text { Deeply-supervised } \\
\text { CNN }\end{array}$ & $2 \mathrm{D}$ & $77 / 4$ & Whole Prostate Gland (DSC: 0.89 ) & 2017 & [138] \\
\hline $\begin{array}{c}\mathrm{T}_{2} \mathrm{~W}(2 \mathrm{D}) \\
\mathrm{DW}(2 \mathrm{D}) \mathrm{MRI}\end{array}$ & $\mathrm{AE}$ & $2 \mathrm{D}$ & $\begin{array}{l}21 \text { leave- } \\
\text { one-out CV }\end{array}$ & $\begin{array}{l}\text { Prostate Tumor (SBE: } 0.89 \text {, sensitivity: } 91 \% \text {, } \\
\text { specificity: } 88 \% \text { ) }\end{array}$ & 2017 & [139] \\
\hline $\mathrm{T}_{2} \mathrm{~W}$ MRI (2D) & $\begin{array}{l}\text { Holistically-nested } \\
\text { FCN }\end{array}$ & $2 \mathrm{D}$ & $\begin{array}{l}250 \text { five-fold } \\
\qquad \mathrm{CV}\end{array}$ & Whole Prostate Gland (DSC: $\mathbf{0 . 8 9}$, IoU: 0.81) & 2017 & [140] \\
\hline DW MRI (2D) & $\begin{array}{l}\text { Modified UNet with } \\
\text { inception blocks }\end{array}$ & $2 \mathrm{D}$ & $\begin{array}{l}141 \text { four- } \\
\text { fold CV }\end{array}$ & $\begin{array}{c}\text { Whole Prostate Gland (DSC: 0.93), } \\
\text { Transition Zone (DSC: 0.88) }\end{array}$ & 2017 & [141] \\
\hline $\mathrm{T}_{2} \mathrm{~W}$ MRI (2D) & $\begin{array}{l}\text { ConvNet with } \\
\text { mixed residual } \\
\text { connections }\end{array}$ & $3 \mathrm{D}$ & $50 / 30$ & Whole Prostate Gland (DSC: 0.87) & 2017 & [142] \\
\hline
\end{tabular}




\begin{tabular}{|c|c|c|c|c|c|c|}
\hline $\mathrm{T}_{2} \mathrm{~W}$ MRI (2D) & $\begin{array}{c}\text { Stacked Sparse AE } \\
\text { (SSAE) + Sparse } \\
\text { patch matching }\end{array}$ & $2 \mathrm{D}$ & $\begin{array}{c}66 \text { two-fold } \\
\text { CV }\end{array}$ & Whole Prostate Gland (DSC: 0.87) & 2016 & [143] \\
\hline $\mathrm{T}_{2} \mathrm{~W}$ MRI (2D) & VNet & $3 \mathrm{D}$ & $50 / 30$ & Whole Prostate Gland (DSC: 0.87) & 2016 & [72] \\
\hline $\begin{array}{c}\mathrm{T}_{2} \mathrm{~W} \text { MRI } \\
\text { (unspecified) }\end{array}$ & $\begin{array}{c}\text { Stacked } \\
\text { independent } \\
\text { subspace analysis } \\
\text { (ISA) }\end{array}$ & $2 \mathrm{D}$ & $\begin{array}{c}30 \text { leave- } \\
\text { one-out CV }\end{array}$ & Whole Prostate Gland (DSC: $\mathbf{0 . 8 6}$ ) & 2013 & [144] \\
\hline
\end{tabular}

Rectal Cancer

\begin{tabular}{|c|c|c|c|c|c|c|}
\hline $\mathrm{CT}$ & DeepLabV3+ & $2 \mathrm{D}$ & $98 / 63$ & $\begin{array}{l}\text { CTV (DSC: 0.88), Bladder (DSC: 0.90), } \\
\text { Small Intestine (DSC: 0.76), } \\
\text { L Fem. Head (DSC: } 0.93), \\
\text { R Fem. Head (DSC: } 0.93 \text { ) }\end{array}$ & 2020 & {$[27]$} \\
\hline $\mathrm{CT}$ & $\begin{array}{l}\text { CNN with cascaded } \\
\text { atrous convolution } \\
\text { (CAC) and spatial }\end{array}$ & $2 \mathrm{D}$ & $\begin{array}{l}100 \\
\text { five-fold CV }\end{array}$ & Rectal Tumor (DSC: 0.78) & \multirow[t]{2}{*}{2018} & \multirow[t]{2}{*}[145]{} \\
\hline $\mathrm{T}_{2} \mathrm{~W}$ MRI (2D) & $\begin{array}{c}\text { pyramid pooling } \\
\text { module (SPP) }\end{array}$ & & $\begin{array}{c}\quad 70 \\
\text { five-fold CV }\end{array}$ & CTV (DSC: 0.85) & & \\
\hline $\mathrm{CT}$ & $\begin{array}{c}\text { Dilated CNN } \\
\text { (transfer learning } \\
\text { from VGG-16) }\end{array}$ & $2 \mathrm{D}$ & $218 / 60$ & $\begin{array}{c}\text { CTV (DSC: 0.87), Bladder (DSC: 0.93), } \\
\text { L Fem. Head (DSC: 0.92), R Fem. Head (DSC: } \\
\text { 0.92), } \\
\text { Intestine (DSC: } 0.65) \text {, Colon (DSC: } 0.62)\end{array}$ & 2017 & [146] \\
\hline $\begin{array}{c}\mathrm{T}_{2} \mathrm{~W}(2 \mathrm{D}), \\
\mathrm{DW}(2 \mathrm{D}) \mathrm{MRI}\end{array}$ & Mask R-CNN & $2 \mathrm{D}$ & $\begin{array}{c}293 / 31(+50 \\
\text { external test } \\
\text { patients })\end{array}$ & Lymph Nodes (DSC: 0.81) & 2020 & [147] \\
\hline $\mathrm{T}_{2} \mathrm{~W}$ MRI (2D) & $\begin{array}{l}\text { CNN (transfer } \\
\text { learning from } \\
\text { ResNet50) }\end{array}$ & $2 \mathrm{D}$ & $461 / 107$ & Rectal Tumor (DSC: 0.82) & 2019 & [148] \\
\hline $\mathrm{T}_{2} \mathrm{~W}$ MRI (3D) & UNet & $2 \mathrm{D}$ & $\begin{array}{l}93 \text { ten-fold } \\
\qquad \mathrm{CV}\end{array}$ & Rectal GTV (DSC: 0.74, IoU: 0.60) & 2018 & [149] \\
\hline $\mathrm{T}_{2} \mathrm{~W}$ MRI (2D) & $\begin{array}{c}\text { FCN (transfer } \\
\text { learning from VGG- } \\
16)\end{array}$ & $2 \mathrm{D}$ & $410 / 102$ & Rectal Tumor (DSC: 0.84) & 2018 & {$[23]$} \\
\hline $\mathrm{T}_{2} \mathrm{~W}$ MRI (2D) & $\begin{array}{l}\text { Hybrid loss FCN } \\
\text { (HL-FCN) }\end{array}$ & $3 \mathrm{D}$ & $\begin{array}{l}64 \text { four-fold } \\
\qquad \mathrm{CV}\end{array}$ & Rectal Tumor (DSC: 0.72) & 2018 & [150] \\
\hline
\end{tabular}




\begin{tabular}{c|c|c|c|c|c|}
\hline $\mathrm{T}_{2} \mathrm{~W}$ & & & & & \\
(unspecified), & CNN & 2D & $70 / 70$ & Rectal Tumor (DSC: 0.69) & 2017 \\
DW (2D) MRI & & & & & [151] \\
\hline
\end{tabular}

Abbreviations: AE, Auto-Encoder; AUC, Area under the ROC Curve; CT, Computed Tomography; CTV, Clinical Target Volume; CV, Cross-Validation; DCE, Dynamic Contrast-Enhanced; DSC, Dice Similarity Coefficient; DWI, Diffusion-Weighted Imaging; GTV, Gross Tumor Volume; LSTM, Long-Short-Term Memory; MRI, Magnetic Resonance Imaging; SBE: Section-Based Evaluation.

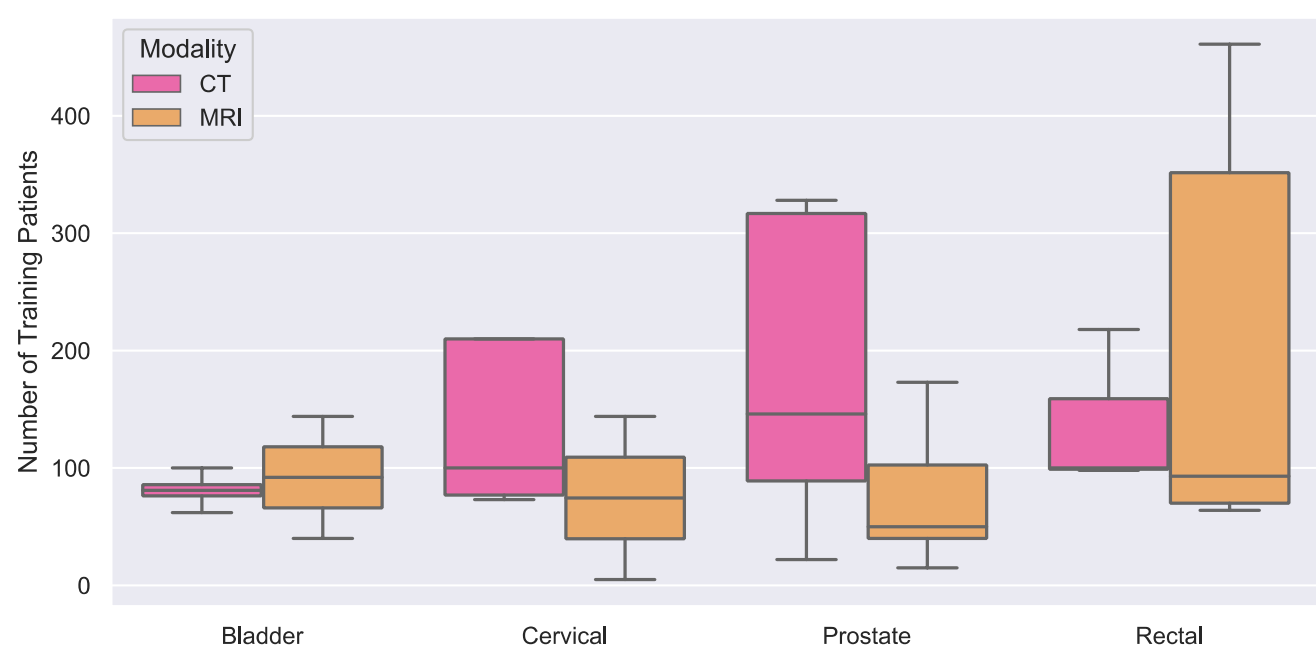

Figure 3. Boxplot of number of training patients used in segmentation applications for bladder (CT studies :4, MRI studies: 2), cervical (CT:2, MRI:5), prostate (CT:12, MRI:40) and rectal (CT:2, MRI:6, CT/MRI:1) cancers. The average number of training patients was 165 from the 74 reviewed studies. The outliers were excluded from this figure for visualization purposes.

\subsection{Cervical Cancer}

Segmentation of cervical tumors remains a challenging task due to large geometrical variations in patient populations and indistinctive soft-tissue boundaries. Previous studies have reported the utility of DW-MRI and ADC for cervical cancer staging, histological grading and nodal status evaluations [152]. Although there is growing interest in quantitative assessment of tumors in radiology, to date, only one previous study by Lin et al. [96] incorporated the use of DL for automatic segmentation and radiomic feature extractions of cervical tumors from ADC maps. The authors demonstrated that their UNetbased segmentation framework outperformed previous ML techniques by a factor of two, potentially providing clinicians with an automated tool to minimize tumor delineation (GTV equivalent) discrepancies. Moreover, Breto et al. [97] developed a Mask R-CNN framework for automatic segmentation of OARs and GTVs for MR-only RT treatment planning for patients with locally advanced cervical cancer. The authors reported that whilst the generated contours for cervix, rectum, bladder, uterus, femur and sigmoid were in good agreement with expert MS, their network underperformed for segmenting smaller and less distinctive soft-tissue structures such as the vagina, parametrium and the mesorectum. However, their results were only based on 5 test patients and not clinically validated. The considerable segmentation complexities in cervical cancer as well as lack of high-quality and annotated databases may have also contributed to the low numbers of studies for DL-based segmentation of cervical tumors on MRI (Table 1).

In the RT literature, Wang et al. [92] proposed a 3D UNet model for CTV (which typically encompasses the tumor, cervix, uterus, ovaries and parametria) and OAR delineations on CT from 25 patients, and suggested that their automatic contours were as accurate as MS performed by a clinical resident with 8 months experience. Liu et al. [153] developed a 3D UNet-based architecture for segmentation of OARs and reported that over $90 \%$ 
of their generated contours were 'highly acceptable' for RT planning through expert oncologist evaluation ( $>15$ years of experience). However, this network underperformed for CTV delineations. In a later study, the authors developed a dual-path UNet network (DpnUNet) consisting of more hidden layers in order to make it more suitable for CTV segmentations where tissue boundaries are unclear. However, despite promising segmentation results their framework was only evaluated on patient scans from a single institution. In contrast, Rhee et al. [94] used a VNet model to generate CT treatment plans and reported that their algorithm achieved on average $80 \%, 97 \%$ and $90 \%$ clinical acceptance rate for primary CTVs, OARs and bony structures respectively. Their framework was validated on 30 cervical cancer patients scanned across three hospitals. The list of the publications for cervical cancer segmentation studies is shown in Table 1.

\subsection{Prostate Cancer}

Previous review studies have investigated various automatic segmentation approaches. However, only one previous study published by Almeida and Tavares [11] provided a systematic review of advances in prostate segmentation which included 28 publications for studies until 2019 (CT: 9, MRI: 19). This study provides an up-to-date review of 52 publications on prostate and/or prostate cancer segmentation (CT: 12, MRI: 40) (see Table 1). Based on our literature search, it is apparent that in recent years the clinical attention for segmentation of the prostate cancer has gravitated towards MRI due to its unparalleled soft-tissue contrast. There remains limited literature for automatic segmentation of the prostate cancer itself, in part because of the technical challenges imposed by the relatively small size of the tumors, background changes with the prostate gland, also because major treatments (e.g. RT) are usually directed towards the whole prostate gland rather than the focal disease. However, as automated decision support tools for prostate cancer diagnosis in MRI are being developed, together with internal radiation boost for prostate cancer and other focal therapies become more widely used, prostate cancer segmentation will become increasingly important.

At present, whole prostate gland (WG), central gland (CG), transition zone (TZ) and peripheral zone (PZ) segmentations have been developed to aid disease assessment ad prostate cancer staging [154]. WG segmentation is also the basis for RT planning. Earlier prostate zonal segmentation algorithms included active appearance [155], continuous max-flow [156] and C-means algorithms [157]. However, these techniques failed to generalize to patient populations from multiple institutions. Due to high clinical demand and technology advancement, DL rapidly found its way into prostate segmentation research. Amongst the MRI-based prostate segmentation studies in our review, 33 studies performed segmentation of WG. However, from these publications only 8 studies also investigated CG, TZ and PZ segmentations [109,114,115,119-121,128,141]. It was observed in these studies that WG segmentation accuracy was superior to PZ and TZ due to large anatomical variations and indistinguishable soft-tissue boundaries. Additionally, only 4 studies provided results on prostate cancer segmentation on MRI [111,119,128,139] (see Table 1).

From the 40 reviewed MRI-based prostate segmentation publications, 32 and 4 used 2D and 3D imaging data for training their DL networks respectively, whilst one study used a combination of 2D and 3D input MRI to train their segmentation algorithms. Additionally, the MR imaging acquisition mode was unspecified for one or all MRI contrasts in 3 studies. Although using volumetric images for training incorporates vital spatial information for organs, it requires considerable computational resources to facilitate training. One advantage of training DL algorithms with 2D convolutional kernels is the ability to use knowledge transfer (transfer learning) from previous models trained on natural images in order to achieve greater segmentation performance. Tian et al. [24] proposed a variant of FCN called PSNet, and through transfer learning achieved satisfactory results. Zhu et al. [138] developed a CNN with deep supervision to better capture multi-level 
feature maps. Attempting to investigate the performance of generative adversarial networks (GANs), Birbiri et al. [110] proposed a conditional GAN (cGAN) and reported that their algorithm with a UNet generator outperformed the standalone UNet model. On the other hand, benefiting from volumetric model training, Milletari et al. [72] developed a 3D CNN called VNet to perform prostate gland segmentation. Feng et al. [131] used a multi-task FCN for training in a semi-supervised manner to overcome lack of adequate training data. Zhu et al. [112] proposed a boundary-weighted strategy to enforce feature learning at the base and apex in prostate from a limited training dataset.

The considerable difficulty in automatic delineation of pelvic organs have inspired the introduction of various segmentation challenges. These include PROMISE12 [158], ASPS13 [159] and PROSTATEx [160]. Amongst the reviewed articles in this study, 28 publications used public datasets for network training and/or validation. For example, Yu et al. [161] developed a 3D CNN with mixed long and short residual connections that enabled high training efficiency and superior feature learning capability from small training datasets. This framework outperformed other proposed algorithms in the PROMISE12 challenge in 2018. Moreover, Brosch et al. [133] developed a framework containing regression-based boundary detection and CNN-based prediction of the distance between a surface mesh and its associated boundary point which ranked first place in the PROMISE12 challenge in 2019. Geng et al. [118] proposed an encoder-decoder architecture with dense dilated pyramidal pooling, and after validating their technique on PROMISE12 and ASPS13 datasets reported that their framework outperformed the then state-the-of-art algorithms for segmentation. Dai et al. [111] developed a region-based CNN (Mask R-CNN) and suggested that their approach was able to perform end-to-end segmentation of the prostate as well as the highly suspicious lesions from the PROSTATEx repository. Based on our literature research, it is evident that the introduction of segmentation challenges along with public and annotated databases for prostate cancer have encouraged research from the wider ML community. The list of available databases and publications for prostate segmentation are shown in Table 2.

Table 2. Public datasets available for prostate cancer segmentation along with the studies that their results were evaluated on these databases. T1W: T1-weighted; $T_{2}$ W: T2-weighted; DW: Diffusion-weighted; PDW: Proton density-weighted; DCE: Dynamic contrastenhanced; MRSI: Magnetic resonance spectroscopic imaging.

\begin{tabular}{|c|c|c|c|c|c|}
\hline Dataset & $\begin{array}{c}\text { Image Modality } \\
\text { (MRI Acquisition } \\
\text { Mode) }\end{array}$ & $\begin{array}{c}\text { Number of } \\
\text { Patients }\end{array}$ & $\begin{array}{c}\text { Ground-Truth } \\
\text { Contours }\end{array}$ & URL & Studies \\
\hline $\begin{array}{c}\text { PROMISE12 } \\
\text { [158] }\end{array}$ & $\mathrm{T}_{2} \mathrm{~W}$ MRI (2D) & 80 & Whole Prostate Gland & $\begin{array}{c}\frac{\text { https://promise12.grand- }}{\text { challenge.org/ }}\end{array}$ & $\begin{array}{l}{[24,108,110,112,} \\
113,117,118,122, \\
124-127,130,135- \\
137,141,142,162]\end{array}$ \\
\hline I2CVB [163] & $\begin{array}{l}\mathrm{T}_{2} \mathrm{~W}(2 \mathrm{D} / 3 \mathrm{D}) \\
\text { DW (2D), } \\
\text { DCE (3D), } \\
\text { MRSI (3D) MRI }\end{array}$ & 40 & $\begin{array}{c}\text { Whole Prostate Gland, } \\
\text { Peripheral Zone, } \\
\text { Central Gland, } \\
\text { Prostate Tumor }\end{array}$ & https://i2cvb.github.io/ & $\begin{array}{c}{[109,119,128,132,} \\
134,164]\end{array}$ \\
\hline BWH [165] & $\begin{array}{l}\mathrm{T}_{1} \mathrm{~W}(2 \mathrm{D} / 3 \mathrm{D}), \\
\mathrm{T}_{2} \mathrm{~W}(2 \mathrm{D}) \mathrm{MRI}\end{array}$ & 230 & Whole Prostate Gland & $\underline{\text { https://prostatemrimagedatabase.com/ }}$ & {$[112,125]$} \\
\hline
\end{tabular}




\begin{tabular}{|c|c|c|c|c|c|}
\hline ASPS13 [159] & $\begin{array}{c}\mathrm{T}_{1} \mathrm{~W}(2 \mathrm{D}), \\
\mathrm{T}_{2} \mathrm{~W}(2 \mathrm{D}), \\
\mathrm{DCE}(3 \mathrm{D}) \mathrm{MRI}\end{array}$ & 156 & $\begin{array}{c}\text { Whole Prostate Gland, } \\
\text { Peripheral Zone }\end{array}$ & $\begin{array}{l}\text { https://wiki.cancerimagingarchive.net/ } \\
\underline{\text { display/Public/NCI- }} \\
\underline{\text { ISBI+2013+Challenge+- }} \\
\frac{+ \text { Automated+Segmentation+of+Prostat }}{\underline{\mathrm{e}+\text { Structures }}}\end{array}$ & {$[24,108,117,118]$} \\
\hline $\begin{array}{c}\text { PROSTATEx } \\
\text { [160] }\end{array}$ & $\begin{array}{c}\mathrm{T}_{2} \mathrm{~W}(2 \mathrm{D}), \\
\mathrm{DW}(2 \mathrm{D}), \\
\text { PDW (3D), } \\
\text { DCE (3D) MRI }\end{array}$ & $\begin{array}{l}330 \\
\text { (malignant } \\
\text { lesions: } 76 \text {, } \\
\text { benign } \\
\text { lesions: } 245 \text { ) }\end{array}$ & Prostate Tumor & https://prostatex.grand-challenge.org/ & {$[114,119,121,123]$} \\
\hline
\end{tabular}

PROMISE12: MICCAI Grand Prostate MR Image Segmentation 2012; I2CVB: Initiative for Collaborative Computer Vision Benchmarking; BWH: The Brigham and Women's Hospital Database; ASPS13: NCI-ISBI 2013 Challenge for Automatic Segmentation of Prostate Structures; PROSTATEx: SPIE-AAPM-NCI Prostate MR Classification Challenge.

Traditionally, OARs and segmentation for RT planning in prostate cancer were performed using volumetric deformable model surface [166], organ-specific modelling [167] and atlas-based techniques [67]. However, contouring through these techniques were poor for patients with abnormal anatomy and data from external institutions, hence hindering the possibility of their integration for online adaptive treatments. Therefore, recent studies have employed DL-based algorithms to develop more efficient, generalizable and consistent segmentation pipelines. The current RT planning workflow uses CT for ROI contouring and radiation dose estimations. Hence, despite poor soft-tissue contrast, segmentation on CT remains desirable. Ma et al. [26] proposed a framework combining a 2D $\mathrm{CNN}$ with multi-atlas label fusion to segment ROIs on CT. To enhance algorithm feature learning capability, Balagopal et al. [106] used a 2D-3D hybrid UNet model containing aggregated residual networks (ResNeXt) and achieved an average DSC of 0.9. However, this was only based on ground-truth data defined by one expert. Wang et al. [101] proposed a 3D FCN with boundary sensitive representations for enhanced organ-specific feature learning and verified their results based on data from 313 patients acquired from multiple CT scanners. On the other hand, Dong et al. [168] used a Cycle Generative Adversarial Network (Cycle-GAN) to generate synthetic MRI from CT to enhance their algorithm's soft-tissue learning capability. However, the impact of registration for contour propagations from MRI to CT was not reported. MRI-only RT planning was also proposed to mitigate these geometrical uncertainties. To the best of our knowledge, there are no public CT databases for prostate segmentation and RT planning.

\subsection{Rectal Cancer}

MRI is the technique of choice for the diagnosis and preoperative staging of rectal cancer [171]. MRI is more accurate in the diagnosis, staging and treatment planning of rectal cancer compared with $\mathrm{CT}$, and also provides quantitative tumor assessment, which can inform treatment response assessment and disease outcomes [170]. Although in recent years numerous studies were published for automatic contouring of pelvic tumors [94,171-174], only a few were reported to address rectal cancer $[27,146,175]$. Based on our article search, 9 studies incorporated DL for rectal cancer segmentation applications (CT: 2, MRI: 6, MRI/CT: 1) (Table 1). Trebeschi et al. [151] published the first CNN-based rectal tumor segmentation study on multi-parametric MRI. Their framework included classification of fixed patches and segmentation of the identified voxels. Although this approach was designed to reduce image redundancy, it ignored context information which adversely affected their network's generalizability in cross-institution model evaluations. Huang et al. [150] developed a volumetric hybrid loss fully-convolutional network (HL- 
FCN) that used Dice-based loss to overcome class imbalance in their training data, however their results were not clinically evaluated. Jian et al. [23] proposed an FCN-based segmentation framework and used transfer learning (VGG-16) to outperform the conventional UNet architecture for rectal tumor segmentation on MRI. Similarly, Wang et al. [148] deployed an FCN model from a pre-trained ResNet50 model to enrich hierarchical feature extraction during network training. The authors evaluated their results on 107 patients from four centers and reported that their network was superior than UNet for tumor contouring. Unfortunately, due to shortage of public databases, direct and meaningful comparison of these algorithms for rectal cancer segmentation remains a challenging task.

To date, only three studies were published on uses of DL for rectal cancer RT treatment planning on CT images. Men et al. [146] proposed a 2D CNN with dilated convolutions and suggested that their network outperformed the traditional UNet architecture. However, the authors reported that their model failed to accurately perform colon and intestine segmentations due to large inter-patient anatomical variabilities and inhomogeneous distribution of the contrast material and gas in these structures. Song et al. [27] investigated DeepLabv3+ and ResUNet architectures for OARs and CTV segmentations and suggested that whilst automatic contouring using these models outperformed the framework proposed by Men et al. [146], they offered different advantages for feature extraction and contouring of pelvic structures. Whilst ResUNet was reported to be an effective algorithm for segmenting visually distinctive structures (e.g. femoral heads, bones), DeepLabv3+ achieved superior segmentation performances for soft-tissues with unclear boundaries (e.g. bladder/small intestine). Their results were in line with a later study by Men et al. [145] who employed cascaded convolutions along with spatial pyramid pooling (SPP) to enhance CTV delineations. However, both of these techniques were based on 2D training which disregards the inter-slice spatial information of OARs and tumor volumes for training.

\section{Discussion}

MRI is increasingly used for the diagnosis, staging and treatment response evaluations of pelvic cancers. Owning to the remarkable advances in imaging technologies and computer processing hardware, imaging diagnostics for cancer disease characterization, treatment assessment and patient follow-up are evolving. Quantitative imaging techniques are showing promise in providing information that can enhance the understanding of diseases and support patient care. For instance, multi-parametric MRI that combines one or more functional MR sequences is now widely used for pelvic tumors. In recent years, DW-MRI has been widely regarded as a reliable quantitative imaging technique that can provide more sensitive disease detection and for the early assessment of treatment response [176]. However, DW-MRI suffers from geometric distortions due to the use of an echo-planar imaging (EPI) sequence which hinders its use for RT planning. With recent introduction of magnetic resonance fingerprinting (MRF) [177], simultaneous quantitative assessment of tissues on MRI without these geometric limitations may eventually become viable. Radiomics, which aims to provide additional insight from scan images that may not be quantified using conventional assessments, has shown potential in directing distinct imaging phenotypes as indicators for biological behavior, therapeutic responses and treatment outcomes [178]. Accurate segmentation of OARs and the GTV are also vital for effective image-guided RT treatment. These applications demand increasing levels of manual workload for ROI delineations which may also be subject to interand/or intra-operator variabilities [2], thus encouraging the rapid development of computer-assisted and automated technologies.

With recent remarkable rise of $\mathrm{AI}$ and specifically DL, substantial research attention has been directed towards bridging the gap between computer vision and patient care. In this review, we presented an overview of the recent DL-based autosegmentation algorithms used in bladder, cervical, prostate and rectal cancers from 74 studies. While these 
studies were proposed to address the key challenges faced in radiation oncology and radiology, the major limitation with cancer tumor segmentation on MRI remains the difficulty in confidently identifying abnormal structures from healthy tissues due to highly variable inter-patient geometrical appearance and potentially poorly-defined soft-tissue boundaries. CT is widely used as the desired imaging modality for radiation dose estimations and RT treatment planning. However, the inadequate soft-tissue contrast on CT necessitates the concurrent adoption of MRI for enhanced visualization of pelvic structures to improve the accuracy of tumor definition, leading to potential segmentation uncertainties caused by mis-registration. Unfortunately, unlike DL applications for natural images, access to medical images for training and evaluating algorithms is restricted due to patient data privacy and labor-intensive ground-truth contour definitions. Therefore, research motivation and technology development from the wider ML community with limited access to adequately large in-house repositories may be consequently hindered. We demonstrated in this review that, although partially due to higher prostate cancer prevalence and appetite for research, the introduction of grand MRI segmentation challenges and publicly-accessible datasets has played an important role in driving prostate cancer research forward. Regrettably, to the best of our knowledge, there are no public and annotated repositories for other pelvic cancer types (MRI or CT) and RT target volumes. Whilst global and institutional efforts are necessary to initiate public repositories, appropriate quality control and external expert auditing need to be in place to ensure data are of high quality $[179,180]$.

The generalizability of DL algorithms can be enhanced by use of multi-vendor and multi-center patient scans for training, however differences in institutional MR imaging protocols may adversely affect segmentation performances. A wide range of DL-based techniques were reviewed in this study that generally proposed architectural, image processing, multi-parametric data entry, loss functions, transfer learning and adversarial training extensions to FCNs which have become the standard model in medical segmentation applications. Researchers routinely use quantitative segmentation evaluation metrics such as DSC and HD to compare their results with other proposed algorithms. Although it may be tempting to rely on these measures to draw definitive conclusions on an algorithm's performance superiority over another, qualitative assessment of results by experts is necessary to ensure clinical demands are met. Moreover, contour definition by experts (radiologist vs. radiation oncologist) with varying clinical experience and the source of training data (single institution vs. multi-center) are other contributing factors to variabilities in ground-truth ROI delineations which can hamper segmentation performance. Some studies incorporated qualitative evaluations to assess the clinical acceptance rate of generated contours [96], however this step is not yet widely undertaken for most pelvic cancer segmentation applications. Based on the reviewed articles, the MRI acquisition mode (2D or 3D) for 5 studies were labelled as 'unspecified' since insufficient acquisition information was provided for training MR images. Furthermore, lack of external validation on public datasets provides a considerable challenge in accurately comparing various algorithms for segmentation applications.

In conclusion, DL in the eyes of clinicians is still seen as a "black box algorithm" due to its limited interpretability for predicted outcome. Therefore, clinical adoption of AIbased frameworks is hindered by their lack of interpretability and explainability when generating inaccurate outcomes. Although DL is a powerful and promising tool for many supervised computer-aided applications, it heavily relies on the quality of input data for training. With absence of standardized and international contouring consensus guidelines to reduce segmentation variabilities, and lack of accessible and annotated public databases, there remains a formidable challenge for true investigation of novel segmentation techniques against existing algorithms. Our review demonstrated the challenges, incentives and public datasets can lead to research contribution from groups from different domains and considerable advancements in technology. Lastly, whilst embracing the exciting future of DL as a catalyst for paradigm shift in disease detection, characterization and treatment planning, researchers and clinicians should be aware of the current 
shortcomings and requirements of automatic pelvic segmentation algorithms in order to push the boundaries of $\mathrm{AI}$ in healthcare.

Author Contributions: Conceptualization, R.K., J.M.W, D.M.K; Writing-Original Draft Preparation, R.K.; Supervision, G.L., J.M.W., C.M., S.L., M.D.B., D.M.K. All authors have read and agreed to the published version of the manuscript.

Funding: This project represents independent research funded by the National Institute for Health Research (NIHR) Biomedical Research Centre and the Clinical Research Facilities at The Royal Marsden NHS Foundation Trust and the Institute of Cancer Research, London, United Kingdom. The views expressed are those of the author(s) and not necessarily those of the NIHR or the Department of Health and Social Care. Gigin Lin received research funding from the Ministry of Science and Technology Taiwan (MOST 110-2628-B-182A-018).

Institutional Review Board Statement: Not applicable.

Informed Consent Statement: Not applicable.

Data Availability Statement: Not applicable.

Conflicts of Interest: The authors declare no conflict of interest. The funders had no role in the design of the review, writing of the manuscript; or the decision to publish.

\section{References}

1. Miles, E.A.; Clark, C.H.; Urbano, M.T.; Bidmead, M.; Dearnaley, D.P.; Harrington, K.J. et al. The impact of introducing intensity modulated radiotherapy into routine clinical practice. Radiother Oncol. 2005; 77 (3): 241-246. Available from: doi: 10.1016/j.radonc.2005.10.011.

2. Nelms, B.E.; Tomé, W.A.; Robinson, G.; Wheeler, James, M.D. Variations in the Contouring of Organs at Risk: Test Case From a Patient With Oropharyngeal Cancer. International journal of radiation oncology, biology, physics; Int J Radiat Oncol Biol Phys. 2012; 82 (1): 368-378. Available from: doi: 10.1016/j.ijrobp.2010.10.019 .

3. Brouwer, C.L.; Steenbakkers, Roel J. H. M.; van den Heuvel E.; Duppen, J.C.; Navran, A.; Bijl, H.P.; et al. 3D Variation in delineation of head and neck organs at risk. Radiation oncology (London, England); Radiat Oncol. 2012; 7 (1): 32 . Available from: doi: 10.1186/1748-717X-7-32 .

4. Boldrini, L.; Cusumano, D.; Cellini, F.; Azario, L.; Mattiucci, G.C.; Valentini, V. Online adaptive magnetic resonance guided radiotherapy for pancreatic cancer: state of the art, pearls and pitfalls. Radiation oncology (London, England); Radiat Oncol. 2019; 14 (1): 71. Available from: doi: 10.1186/s13014-019-1275-3 .

5. Mikeljevic, J.S.; Haward, R.; Johnston, C.; Crellin, A.; Dodwell, D., Jones, A. et al. Trends in postoperative radiotherapy delay and the effect on survival in breast cancer patients treated with conservation surgery. Br J Cancer. 2004; 90 (7): 1343-1348. Available from: doi: 10.1038/sj.bjc.6601693 .

6. Chen, Z.; King, W.; Pearcey, R.; Kerba, M.; Mackillop, W.J. The relationship between waiting time for radiotherapy and clinical outcomes: A systematic review of the literature. Radiother Oncol. 2007; 87 (1): 3-16. Available from: doi: 10.1016/j.radonc.2007.11.016 .

7. Hesamian, M.H.; Jia, W.; He, X.; Kennedy, P. Deep Learning Techniques for Medical Image Segmentation: Achievements and Challenges. J Digit Imaging. 2019; 32 (4): 582-596. Available from: doi: 10.1007/s10278-019-00227-x .

8. Cardenas, C.E.; Yang, J.; Anderson, B.M.; Court, L.E.; Brock, K.B. Advances in Auto-Segmentation. Semin Radiat Oncol. 2019; 29 (3): 185-197. Available from: doi: 10.1016/j.semradonc.2019.02.001 .

9. Rizwan, I.; Haque, I.; Neubert, J. Deep learning approaches to biomedical image segmentation. Informatics in medicine unlocked. 2020; 18 100297. Available from: doi: 10.1016/j.imu.2020.100297.

10. Zhou, T.; Ruan, S.; Canu, S. A review: Deep learning for medical image segmentation using multi-modality fusion. Array. 2019; 3-4 100004. Available from: doi: 10.1016/j.array.2019.100004.

11. Almeida, G.; Tavares, J.M.R.S. Deep Learning in Radiation Oncology Treatment Planning for Prostate Cancer: A Systematic Review. Journal of medical systems. 2020; 44 (10): 179. Available from: doi: 10.1007/s10916-020-01641-3 .

12. Ueda, D.; Shimazaki, A.; Miki, Y. Technical and clinical overview of deep learning in radiology. Jpn J Radiol. 2019; 37 (1): 15-33. Available from: doi: 10.1007/s11604-018-0795-3 .

13. Boldrini, L.; Bibault, J.; Masciocchi, C.; Shen, Y.; Bittner, M. Deep Learning: A Review for the Radiation Oncologist. Frontiers in oncology. 2019; 9,977. Available from: doi: 10.3389/fonc.2019.00977 . 
14. Meyer, P.; Noblet, V.; Mazzara, C.; Lallement, A. Survey on deep learning for radiotherapy. Computers in biology and medicine; Comput Biol Med. 2018; 98 126-146. Available from: doi: 10.1016/j.compbiomed.2018.05.018 .

15. Kowalski, R. Computational Logic and Human Thinking: How to Be Artificially Intelligent. Cambridge: Cambridge University Press; 2011.

16. Hebb, D.O. The organization of behavior: A neuropsychological theory. Psychology Press; 1949.

17. McCulloch, W.S.; Pitts, W. A logical calculus of the ideas immanent in nervous activity. 5(4) ed.: The bulletin of mathematical biophysics; 1943, 5, 115-133. Available from: doi: 10.1007/BF02478259

18. Rosenblatt, F. The perceptron: A probabilistic model for information storage and organization in the brain. Psychol Rev. 1958; 65 (6): 386-408. Available from: doi: 10.1037/h0042519.

19. Rumelhart, D.E.; Hinton, G.E.; Williams, R.J. Learning representations by back-propagating errors. Nature. 1986; 323 (6088): 533 536. Available from: doi: $10.1038 / 323533 a 0$.

20. Lecun, Y.; Bottou, L.; Bengio, Y.; Haffner, P. Gradient-based learning applied to document recognition. Proceedings of the IEEE. 1998; 86 (11): 2278-2324. Available from: doi: 10.1109/5.726791 .

21. Goodfellow, I.; Bengio, Y.; Courville, A. Deep learning. Cambridge, Massachusetts ;: The MIT Press; 2016.

22. Zhang, Q.; Zhu, S. Visual interpretability for deep learning: a survey. Frontiers of information technology E electronic engineering. 2018; 19 (1): 27-39. Available from: doi: 10.1631/FITEE.1700808 .

23. Jian, J.; Xiong, F.; Xia, W.; Zhang, R.; Gu, J.; Wu, X. et al. Fully convolutional networks (FCNs)-based segmentation method for colorectal tumors on T2-weighted magnetic resonance images. Australas Phys Eng Sci Med. 2018; 41 (2): 393-401.

24. Tian, Z.; Liu, L.; Zhang, Z.; Fei, B. PSNet: prostate segmentation on MRI based on a convolutional neural network. J.Med.Imag. 2018; 5 (2): 021208. Available from: doi: 10.1117/1.JMI.5.2.021208 .

25. Tian, Z.; Liu, L.; Fei, B. Deep convolutional neural network for prostate MR segmentation. International journal for computer assisted radiology and surgery. 2018; 13 (11): 1687-1696. Available from: doi: 10.1007/s11548-018-1841-4 .

26. Ma, L.; Guo, R.; Zhang, G.; Tade, F.; Schuster, D.M.; Nieh, P. et al. Automatic segmentation of the prostate on CT images using deep learning and multi-atlas fusion. Proc SPIE Int Soc Opt Eng. 2017.

27. Song, Y.; Hu, J.; Wu, Q.; Xu, F.; Nie, S.; Zhao, Y. et al. Automatic delineation of the clinical target volume and organs at risk by deep learning for rectal cancer postoperative radiotherapy. Radiotherapy and Oncology. 2020; 145, 186-192. Available from: doi: 10.1016/j.radonc.2020.01.020 .

28. Chai, X.; van Herk, M.; Betgen, A.; Hulshof, M.; Bel, A. Automatic bladder segmentation on CBCT for multiple plan ART of bladder cancer using a patient-specific bladder model. Phys.Med.Biol. 2012; 57 (12): 3945-3962. Available from: doi: 10.1088/00319155/57/12/3945 .

29. Gulliford, S.L.; Webb, S.; Rowbottom, C.G.; Corne, D.W.; Dearnaley, D.P. Use of artificial neural networks to predict biological outcomes for patients receiving radical radiotherapy of the prostate. Radiother Oncol. 2004; 71 (1): 3-12. Available from: doi: 10.1016/j.radonc.2003.03.001 .

30. Kim, D.W.; Lee, S.; Kwon, S.; Nam, W.; Cha, I.; Kim, H.J. Deep learning-based survival prediction of oral cancer patients. Sci Rep. 2019; 9 (1): 6994. Available from: doi: 10.1038/s41598-019-43372-7 .

31. Han, X. MR-based synthetic CT generation using a deep convolutional neural network method. Med Phys. 2017; 44 (4): 14081419. Available from: doi: 10.1002/mp.12155.

32. Nie. D.; Cao, X.; Gao, Y.; Wang, L.; Shen, D. Estimating CT Image from MRI Data Using 3D Fully Convolutional Networks. Deep Learn Data Label Med Appl. 2016; 170-178. Available from: doi: 10.1007/978-3-319-46976-8_18 .

33. Zhen, X.; Chen, J.; Zhong, Z.; Hrycushko, B.; Zhou, L.; Jiang, S. et al. Deep convolutional neural network with transfer learning for rectum toxicity prediction in cervical cancer radiotherapy: a feasibility study. Phys.Med.Biol. 2017; 62 (21): 8246-8263. Available from: doi: 10.1088/1361-6560/aa8d09 .

34. Ma, M.; Kovalchuk, N.; Buyyounouski, M.K.; Xing, L.; Yang, Y. Incorporating dosimetric features into the prediction of 3D VMAT dose distributions using deep convolutional neural network. Phys.Med.Biol. 2019; 64 (12): 125017. Available from: doi: 10.1088/1361-6560/ab2146.

35. Soni, P.; Maturen, K.E.; Prisciandaro, J.I.; Zhou, J.; Cao, Y.; Balter, J. et al. Using MRI to Characterize Small Anatomic Structures Critical to Pelvic Floor Stability in Gynecologic Cancer Patients Undergoing Radiation Therapy. International journal of radiation oncology, biology, physics. 2015; 93 (3): E608. Available from: doi: 10.1016/j.ijrobp.2015.07.2100 .

36. Colosio, A.; Soyer, P.; Rousset, P.; Barbe, C.; Nguyen, F.; Bouché, O. et al. Value of diffusion-weighted and gadolinium-enhanced MRI for the diagnosis of pelvic recurrence from colorectal cancer. J Magn Reson Imaging. 2014; 40 (2): 306-313. Available from: doi: 10.1002/jmri.24366 .

37. Nam, E.J.; Yun, M.J.; Oh, Y.T.; Kim, J.W.; Kim, J.H.; Kim, S. et al. Diagnosis and staging of primary ovarian cancer: Correlation between PET/CT, Doppler US, and CT or MRI. Gynecol Oncol. 2009; 116 (3): 389-394. Available from: doi: 10.1016/j.ygyno.2009.10.059.

38. Fütterer, J.J.; Briganti, A.; De Visschere, P.; Emberton, M.; Giannarini, G.; Kirkham, A. et al. Can Clinically Significant Prostate Cancer Be Detected with Multiparametric Magnetic Resonance Imaging? A Systematic Review of the Literature. European urology; Eur Urol. 2015; 68 (6): 1045-1053. Available from: doi: 10.1016/j.eururo.2015.01.013 .

39. Valerio, M.; Donaldson, I.; Emberton, M.; Ehdaie, B.; Hadaschik, B.A.; Marks, L.S. et al. Detection of Clinically Significant Prostate Cancer Using Magnetic Resonance Imaging-Ultrasound Fusion Targeted Biopsy: A Systematic Review. Eur Urol. 2014; 68 (1): 8-19. Available from: doi: 10.1016/j.eururo.2014.10.026 . 
40. Muller, B.G.; Fütterer, J.J.; Gupta, R.T.; Katz, A.; Kirkham, A.; Kurhanewicz, J. et al. The role of magnetic resonance imaging (MRI) in focal therapy for prostate cancer: recommendations from a consensus panel: Role of MRI in focal therapy for prostate cancer. BJU international. 2014; 113 (2): 218-227. Available from: doi: 10.1111/bju.12243 .

41. Eldred-Evans, D.; Tam, H.; Smith, A.; Winkler, M.; Ahmed, H.U. Use of imaging to optimise prostate cancer tumour volume assessment for focal therapy planning. Curr Urol Rep. 2020, 21(10), 30. Available from: doi: 10.1007/s11934-020-00987-y .

42. Mazaheri, Y.; Hricak, H.; Touijer, K.; Koutcher, J.A.; Fine, S.W.; Akin, O. et al. Prostate Tumor Volume Measurement with Combined T2-weighted Imaging and Diffusion-weighted MR: Correlation with Pathologic Tumor Volume. Radiology. 2009; 252 (2): 449-457. Available from: doi: 10.1148/radiol.2523081423.

43. Carl Jaffe, C. Measures of Response: RECIST, WHO, and New Alternatives. J Clin Oncol. 2006; 24 (20): 3245-3251. Available from: doi: 10.1200/JCO.2006.06.5599.

44. Padhani, A.R.; Liu, G.; Koh D.M.; Chenevert, T.L.; Thoeny, H.C.; Takahara, T. et al. Diffusion-weighted magnetic resonance imaging as a cancer biomarker: consensus and recommendations. Neoplasia. 2009; 11 (2): 102-125.

45. Lin, Y.; Lin, G.; Hong, J.; Lin, Y.; Chen, F.; Ng, S. et al. Diffusion radiomics analysis of intratumoral heterogeneity in a murine prostate cancer model following radiotherapy: Pixelwise correlation with histology: Diffusion Radiomics for Radiotherapy. Journal of magnetic resonance imaging. 2017; 46 (2): 483-489. Available from: doi: 10.1002/jmri.25583 .

46. Schob, S.; Meyer, H.J.; Pazaitis, N.; Schramm, D.; Bremicker, K.; Exner, M. et al. ADC Histogram Analysis of Cervical Cancer Aids Detecting Lymphatic Metastases-a Preliminary Study. Mol Imaging Biol. 2017; 19 (6): 953-962. Available from: doi: 10.1007/s11307-017-1073-y .

47. Lin, G.; Yang, L.; Lin, Y.; Huang, Y.; Liu, F.; Wang, C. et al. Prognostic model based on magnetic resonance imaging, wholetumour apparent diffusion coefficient values and HPV genotyping for stage IB-IV cervical cancer patients following chemoradiotherapy. Eur Radiol. 2019; 29 (2): 556-565. Available from: doi: 10.1007/s00330-018-5651-4 .

48. Thiesse, P.; Ollivier, L.; Di Stefano-Louineau, D.; Négrier, S.; Savary, J.; Pignard, K. et al. Response rate accuracy in oncology trials: reasons for interobserver variability. Groupe Français d'Immunothérapie of the Fédération Nationale des Centres de Lutte Contre le Cancer. Journal of Clinical Oncology. 1997; 15 (12): 3507-3514.

49. Pollard, J.M.; Wen, Z.; Sadagopan, R.; Wang, J.; Ibbott, G.S. The future of image-guided radiotherapy will be MR guided. Br J Radiol. 2017; 90 (1073): 20160667. Available from: doi: 10.1259/bjr.20160667.

50. Chavaudra, J.; Bridier, A. Definition of volumes in external radiotherapy: ICRU reports 50 and 62. Cancer Radiother. 2001; 5 (5): 472.

51. The Royal College of Radiologists, Society of Radiographers, College, Institute of Physics in Medicine, and Engineering. On target: ensuring geometric accuracy in radiotherapy. Technical report, The Royal College of Radiologists. 2008.

52. Chan, T.F.; Vese, L.A. Active contour and segmentation models using geometric PDE's for medical imaging. Geometric methods in bio-medical image processing. Springer; 2002. 63-75.

53. Jiang, X.; Zhang, R.; Nie, S. Image segmentation based on level set method. Physics Procedia. 2012; 33 840-845.

54. Boykov, Y.; Jolly, M. Interactive organ segmentation using graph cuts. International conference on medical image computing and computer-assisted intervention. Springer. 2000. 276-286.

55. Beucher, S. Use of watersheds in contour detection. Proceedings of the International Workshop on Image Processing: CCETT; 1979.

56. Naik, S.; Doyle, S.; Agner, S.; Madabhushi, A.; Feldman, M.; Tomaszewski, J. Automated gland and nuclei segmentation for grading of prostate and breast cancer histopathology. 5th IEEE International Symposium on Biomedical Imaging: From Nano to Macro. 2008. pp. 284-287. Available from: 10.1109/ISBI.2008.4540988.

57. Zyout, I.; Abdel-Qader, I.; Jacobs, C. Bayesian Classifier with Simplified Learning Phase for Detecting Microcalcifications in Digital Mammograms. Int J Biomed Imaging. 2010; 2009 767805-13. Available from: doi: 10.1155/2009/767805.

58. Qiao, J.; Cai, X.; Xiao, Q.; Chen, Z.; Kulkarni, P.; Ferris, C. et al. Data on MRI brain lesion segmentation using K-means and Gaussian Mixture Model-Expectation Maximization. Data in brief. 2019; 27, 104628 . Available from: doi: 10.1016/j.dib.2019.104628 .

59. Zhang, Y.; Brady, M.; Smith, S. Segmentation of brain MR images through a hidden Markov random field model and the expectation-maximization algorithm. IEEE Trans Med Imaging. 2001; 20(1): 45-57. Available from: doi: 10.1109/42.906424 .

60. Iglesias, J.E.; Sabuncu, M.R. Multi-atlas segmentation of biomedical images: A survey. Med Image Anal. $2015 ; 24$ (1): $205-219$. Available from: doi: 10.1016/j.media.2015.06.012 .

61. Blezek, D.J.; Miller, J.V. Atlas stratification. Med Image Anal. 2007; 11(5): 443-457. Available from: doi: 10.1016/j.media.2007.07.001

62. Commowick, O.; Malandain, G. Efficient Selection of the Most Similar Image in a Database for Critical Structures Segmentation. In International Conference on Medical Image Computing and Computer-Assisted Intervention, 203-210. Berlin, Heidelberg: Springer Berlin Heidelberg; 2007.

63. Commowick, O.; Warfield, S.K.; Malandain, G. Using Frankenstein's Creature Paradigm to Build a Patient Specific Atlas. International Conference on Medical Image Computing and Computer-Assisted Intervention. Berlin, Heidelberg: Springer Berlin Heidelberg; 2009.

64. Yang, J.; Amini, A.; Williamson, R.; Zhang, L.; Zhang, Y.; Komaki, R. et al. Automatic contouring of brachial plexus using a multi-atlas approach for lung cancer radiation therapy. Practical radiation oncology. 2013; 3 (4): e139-e147.

65. Sharp, G.; Fritscher, K.D.; Pekar, V.; Peroni, M.; Shusharina, N.; Veeraraghavan, H. et al. Vision 20/20: perspectives on automated image segmentation for radiotherapy. Med phys. 2014; 41 (5) .

66. Harrison, A.; Galvin, J.; Yu, Y.; Xiao, Y. SU-FF-J-172: Deformable Fusion and Atlas Based Autosegmentation: MimVista Vs. CMS Focal ABAS. Med phys. 2009; 36 (6): 2517. Available from: doi: 10.1118/1.3181465 . 
67. La Macchia, M.; Fellin ,F.; Amichetti, M.; Cianchetti, M.; Gianolini, S.; Paola, V. et al. Systematic evaluation of three different commercial software solutions for automatic segmentation for adaptive therapy in head-and-neck, prostate and pleural cancer. Radiation oncology (London, England); Radiat Oncol. 2012; 7 (1): 160. Available from: doi: 10.1186/1748-717X-7-160 .

68. Menzel, H. International Commission on Radiation Units and Measurements. Journal of the ICRU. 2014; 14 (2): 1-2. Available from: doi: 10.1093/jicru/ndx006.

69. Long, J.; Shelhamer, E.; Darrell, T. Fully convolutional networks for semantic segmentation. IEEE; 2015.

70. Ronneberger, O.; Fischer, P.; Brox, T. U-Net: Convolutional Networks for Biomedical Image Segmentation. Cham: Springer International Publishing; 2015.

71. Çiçek, Ö.; Abdulkadir, A.; Lienkamp, S.S.; Brox, T.; Ronneberger, O. 3D U-Net: Learning Dense Volumetric Segmentation from Sparse Annotation. Cham: Springer International Publishing; 2016.

72. Milletari, F.; Navab, N.; Ahmadi, S. V-Net: Fully Convolutional Neural Networks for Volumetric Medical Image Segmentation. IEEE; 2016.

73. Kamnitsas, K.; Ferrante, E.; Parisot, S.; Ledig, C.; Nori, A.V.; Criminisi, A. et al. DeepMedic for brain tumor segmentation. In International workshop on Brainlesion: Glioma, multiple sclerosis, stroke and traumatic brain injuries (pp. 138-149). Springer, Cham.

74. Liang-Chieh, C.; Papandreou, G.; Kokkinos, I.; Murphy, K.; Yuille, A.L. DeepLab: Semantic Image Segmentation with Deep Convolutional Nets, Atrous Convolution, and Fully Connected CRFs. IEEE Trans Pattern Anal Mach Intell. 2018; 40 (4): 834-848. Available from: doi: 10.1109/TPAMI.2017.2699184 .

75. Liu, X.; Song, L.; Liu, S.; Zhang, Y. A Review of Deep-Learning-Based Medical Image Segmentation Methods. Sustainability (Basel, Switzerland). 2021; 13 (1224): 1224. Available from: doi: 10.3390/su13031224 .

76. Dice, L.R. Measures of the Amount of Ecologic Association Between Species. Ecology (Durham). 1945; 26 (3): $297-302$. Available from: doi: 10.2307/1932409.

77. Nikolov, S.; Blackwell, S.; Mendes, R.; De Fauw, J.; Meyer, C.; Hughes, C. et al. Deep learning to achieve clinically applicable segmentation of head and neck anatomy for radiotherapy. arXiv preprint arXiv:1809.04430. 2018; .

78. Ge, F.; Wang, S.; Liu, T. New benchmark for image segmentation evaluation. Journal of electronic imaging. $2007 ; 16$ (3): 33011. Available from: doi: 10.1117/1.2762250 .

79. Huttenlocher, D.P.; Klanderman, G.A.; Rucklidge, W.J. Comparing images using the Hausdorff distance. IEEE Transactions on Pattern Analysis and Machine Intelligence. 1993; 15 (9): 850-863. Available from: doi: 10.1109/34.232073 .

80. Cha, K.H.; Hadjiiski, L.M.; Samala, R.K.; Chan, H.; Cohan, R.H.; Caoili, E.M. et al. Bladder Cancer Segmentation in CT for Treatment Response Assessment: Application of Deep-Learning Convolution Neural Network-A Pilot Study. Tomography. 2016; 2 (4): 421-429. Available from: doi: 10.18383/j.tom.2016.00184.

81. Ma, X.; Hadjiiski, L.M.; Wei, J.; Chan, H.; Cha, K.H.; Cohan, R.H. et al. U-Net based deep learning bladder segmentation in CT urography. Med Phys. 2019; 46 (4): 1752-1765. Available from: doi: 10.1002/mp.13438 .

82. Duan, C.; Yuan, K.; Liu, F.; Xiao, P.; Lv, G.; Liang, Z. An Adaptive Window-Setting Scheme for Segmentation of Bladder Tumor Surface via MR Cystography. IEEE Trans Inf Technol Biomed. 2012; 16 (4): 720-729. Available from: doi: 10.1109/TITB.2012.2200496

83. Duan, C.; Liang, Z.; Bao, S.; Zhu, H.; Wang, S.; Zhang, G. et al. A Coupled Level Set Framework for Bladder Wall Segmentation With Application to MR Cystography. IEEE Trans Med Imaging. 2010; 29 (3): 903-915. Available from: doi: 10.1109/TMI.2009.2039756 .

84. Han, H.; Li, L.; Duan, C.; Zhang, H.; Zhao, Y.; Liang, Z. A unified EM approach to bladder wall segmentation with coupled level-set constraints. Med Image Anal. 2013; 17 (8): 1192-1205. Available from: doi: 10.1016/j.media.2013.08.002 .

85. Qin, X.; Li, X.; Liu, Y.; Lu, H.; Yan, P. Adaptive Shape Prior Constrained Level Sets for Bladder MR Image Segmentation. IEEE J Biomed Health Inform. 2014; 18 (5): 1707-1716. Available from: doi: 10.1109/JBHI.2013.2288935 .

86. Cha, K.H.; Hadjiiski, L.; Samala, R.K.; Chan, H.; Caoili, E.M.; Cohan, R.H. Urinary bladder segmentation in CT urography using deep-learning convolutional neural network and level sets. Med Phys. 2016; 43 (4): 1882-1896. Available from: doi: 10.1118/1.4944498 .

87. Xu, X.; Zhou, F.; Liu, B. Automatic bladder segmentation from CT images using deep CNN and 3D fully connected CRF-RNN. Int J Comput Assist Radiol Surg. 2018; 13 (7): 967-975. Available from: doi: 10.1007/s11548-018-1733-7 .

88. Dolz, J.; Xu, X.; Rony, J.; Yuan, J.; Liu, Y.; Granger, E. et al. Multiregion segmentation of bladder cancer structures in MRI with progressive dilated convolutional networks. Med Phys. 2018; 45 (12): 5482-5493.

89. Li, R.; Chen, H.; Gong, G.; Wang, L. Bladder Wall Segmentation in MRI Images via Deep Learning and Anatomical Constraints. Annu Int Conf IEEE Eng Med Biol Soc. 2020:1629-1632.

90. Liu, Z.; Liu, X.; Xiao, B.; Wang, S.; Miao, Z.; Sun, Y. et al. Segmentation of organs-at-risk in cervical cancer CT images with a convolutional neural network. Phys Med. 2020; 69 184-191. Available from: doi: 10.1016/j.ejmp.2019.12.008 .

91. Liu, Z.; Liu, X.; Guan, H.; Zhen, H.; Sun, Y.; Chen, Q. et al. Development and validation of a deep learning algorithm for autodelineation of clinical target volume and organs at risk in cervical cancer radiotherapy. Radiother Oncol. 2020; 153 172-179. Available from: doi: 10.1016/j.radonc.2020.09.060

92. Wang, Z.; Chang, Y.; Peng, Z.; Lv, Y.; Shi, W.; Wang, F. et al. Evaluation of deep learning-based auto-segmentation algorithms for delineating clinical target volume and organs at risk involving data for 125 cervical cancer patients. J Appl Clin Med Phys. 2020; 21 (12): 272-279. Available from: doi: 10.1002/acm2.13097 .

93. Zhang, D.; Yang, Z.; Jiang, S.; Zhou, Z.; Meng, M.; Wang, W. Automatic segmentation and applicator reconstruction for CTbased brachytherapy of cervical cancer using 3D convolutional neural networks. Journal of Applied Clinical Medical Physics. 2020; 21 (10): 158-169. 
94. Rhee, D.J.; Jhingran, A.; Rigaud, B.; Netherton, T.; Cardenas, C.E.; Zhang, L. et al. Automatic contouring system for cervical cancer using convolutional neural networks. Med Phys. 2020; 47 (11): 5648-5658. Available from: doi: 10.1002/mp.14467 .

95. Breto, A.L.; Zavala-Romero, O.; Asher, D.; Baikovitz, J.B.; Ford, J.; Stoyanova, R. et al. A Deep Learning Pipeline for per-Fraction Automatic Segmentation of GTV and OAR in cervical cancer. Int. J. Radiat. Oncol. Biol. Phys. 2019; 105 (1): S202. Available from: doi: 10.1016/j.ijrobp.2019.06.267 .

96. Lin, Y.; Lin, C.; Lu, H.; Chiang, H.; Wang, H.; Huang, Y. et al. Deep learning for fully automated tumor segmentation and extraction of magnetic resonance radiomics features in cervical cancer. Eur Radiol. 2020; 30 (3): 1297-1305. Available from: doi: 10.1007/s00330-019-06467-3 .

97. Wong, J.; Fong, A.; McVicar, N.; Smith, S.; Giambattista, J.; Wells, D. et al. Comparing deep learning-based auto-segmentation of organs at risk and clinical target volumes to expert inter-observer variability in radiotherapy planning. Radiother Oncol. 2020; 144 152-158. Available from: doi: 10.1016/j.radonc.2019.10.019.

98. Kiljunen, T.; Akram, S.; Niemelä, J.; Löyttyniemi, E; Seppälä, J.; Heikkilä J, et al. A Deep Learning-Based Automated CT Segmentation of Prostate Cancer Anatomy for Radiation Therapy Planning-A Retrospective Multicenter Study. Diagnostics (Basel). 2020; 10 (11): 959. Available from: doi: 10.3390/diagnostics10110959 .

99. Zhou, S.; Nie, D.; Adeli, E.; Yin, J.; Lian, J.; Shen, D. High-Resolution Encoder-Decoder Networks for Low-Contrast Medical Image Segmentation. IEEE Trans Image Process. 2019; 29 461-475. Available from: doi: 10.1109/TIP.2019.2919937 .

100. Dong, X.; Lei, Y.; Tian, S.; Wang, T.; Patel, P.; Curran, W.J. et al. Synthetic MRI-aided multi-organ segmentation on male pelvic CT using cycle consistent deep attention network. Radiother Oncol. 2019; 141 192-199. Available from: doi: 10.1016/j.radonc.2019.09.028 .

101. Wang, S.; He, K.; Nie, D.; Zhou, S.; Gao, Y.; Shen, D. CT male pelvic organ segmentation using fully convolutional networks with boundary sensitive representation. Med Image Anal. 2019; 54 168-178. Available from: doi: 10.1016/j.media.2019.03.003 .

102. Liu, C.; Gardner, S.J.; Wen, N.; Elshaikh, M.A.; Siddiqui, F.; Movsas, B. et al. Automatic Segmentation of the Prostate on CT Images Using Deep Neural Networks (DNN). Int J Radiat Oncol Biol Phys. 2019; 104 (4): 924-932. Available from: doi: 10.1016/j.ijrobp.2019.03.017.

103. Kearney, V.; Chan, J.W.; Wang, T.; Perry, A.; Yom, S.S.; Solberg, T.D. Attention-enabled 3D boosted convolutional neural networks for semantic CT segmentation using deep supervision. Phys.Med.Biol. 2019; 64 (13): 135001. Available from: doi: 10.1088/1361-6560/ab2818.

104. He, K.; Cao, X.; Shi, Y.; Nie, D.; Gao, Y.; Shen, D. Pelvic Organ Segmentation Using Distinctive Curve Guided Fully Convolutional Networks. IEEE Trans Med Imaging. 2019; 38 (2): 585-595. Available from: doi: 10.1109/TMI.2018.2867837 .

105. Kazemifar, S.; Balagopal, A.; Nguyen, D.; McGuire, S.; Hannan, R.; Jiang, S. et al. Segmentation of the prostate and organs at risk in male pelvic CT images using deep learning. Biomed.Phys.Eng.Express. 2018; 4 (5): 55003. Available from: doi: 10.1088/20571976/aad100.

106. Balagopal, A.; Kazemifar, S.; Nguyen, D.; Lin, M.; Hannan, R.; Owrangi, A. et al. Fully automated organ segmentation in male pelvic CT images. Phys.Med.Biol. 2018; 63 (24): 245015. Available from: doi: 10.1088/1361-6560/aaf11c .

107. Shi, Y.; Yang, W.; Gao, Y.; Shen, D. Does Manual Delineation only Provide the Side Information in CT Prostate Segmentation? Medical Image Computing and Computer Assisted Intervention. 2017; 692-700. Available from: doi: 10.1007/978-3-319-66179-7_79 .

108. Jia, H.; Xia, Y.; Song, Y.; Zhang, D.; Huang, H.; Zhang, Y. et al. 3D APA-Net: 3D Adversarial Pyramid Anisotropic Convolutional Network for Prostate Segmentation in MR Images. IEEE Trans Med Imaging. 2020; 39 (2): 447-457. Available from: doi: 10.1109/TMI.2019.2928056

109. Khan, Z.; Yahya, N.; Alsaih, K.; Meriaudeau, F. Segmentation of Prostate in MRI Images Using Depth Separable Convolution Operations. International Conference on Intelligent Human Computer Interaction: Springer; 2020; 132-141.

110. Cem Birbiri, U., Hamidinekoo, A.; Grall, A.; Malcolm, P; Zwiggelaar, R. Investigating the Performance of Generative Adversarial Networks for Prostate Tissue Detection and Segmentation. Journal of imaging. 2020; 6 (9): 83. Available from: doi: 10.3390/jimaging6090083.

111. Dai, Z.; Carver, E.; Liu, C.; Lee, J.; Feldman, A.; Zong, W. et al. Segmentation of the Prostatic Gland and the Intraprostatic Lesions on Multiparametic Magnetic Resonance Imaging Using Mask Region-Based Convolutional Neural Networks. Advances in radiation oncology. 2020; 5 (3): 473-481. Available from: doi: 10.1016/j.adro.2020.01.005 .

112. Zhu, Q.; Du, B.; Yan, P. Boundary-Weighted Domain Adaptive Neural Network for Prostate MR Image Segmentation. IEEE Trans Med Imaging. 2020; 39 (3): 753-763. Available from: doi: 10.1109/TMI.2019.2935018 .

113. Tian, Z.; Li, X.; Zheng, Y.; Chen, Z.; Shi, Z.; Liu, L. et al. Graph-convolutional-network-based interactive prostate segmentation in MR images. Med phys. 2020; 47 (9): 4164-4176.

114. Aldoj, N.; Biavati, F.; Michallek, F.; Stober, S.; Dewey, M. Automatic prostate and prostate zones segmentation of magnetic resonance images using DenseNet-like U-net. Sci Rep. 2020; 10 (1): 14315. Available from: doi: 10.1038/s41598-020-71080-0 .

115. Rundo, L.; Han, C.; Zhang, J.; Hataya, R.; Nagano, Y.; Militello, C. et al. CNN-Based Prostate Zonal Segmentation on T2Weighted MR Images: A Cross-Dataset Study. Singapore: Springer Singapore; 2019.

116. Savenije, M.H.F.; Maspero, M.; Sikkes, G.G.; van der Voort van Zyp, Jochem,R.N.; T J Kotte, A.N., Bol G.H. et al. Clinical implementation of MRI-based organs-at-risk auto-segmentation with convolutional networks for prostate radiotherapy. Radiat Oncol. 2020; 15 (1): 104. Available from: doi: 10.1186/s13014-020-01528-0 .

117. Lu, Z.; Zhao, M.; Pang, Y. CDA-Net for Automatic Prostate Segmentation in MR Images. Applied sciences. $2020 ; 10$ (6678): 6678. Available from: doi: 10.3390/app10196678 .

118. Geng, L.; Wang, J.; Xiao, Z.; Tong, J.; Zhang, F.; Wu, J. Encoder-decoder with dense dilated spatial pyramid pooling for prostate MR images segmentation. Comput Assist Surg. 2019; 24 13-19. Available from: doi: 10.1080/24699322.2019.1649069. 
119. Liu, Z.; Jiang, W.; Lee, K.; Lo, Y.; Ng, Y.; Dou, Q., et al. A Two-Stage Approach for Automated Prostate Lesion Detection and Classification with Mask R-CNN and Weakly Supervised Deep Neural Network. In Workshop on Artificial Intelligence in Radiation Therapy. Springer, Cham. 2019; 43-51.

120. Zabihollahy, F.; Schieda, N.; Krishna Jeyaraj, S.; Ukwatta, E. Automated segmentation of prostate zonal anatomy on T2weighted (T2W) and apparent diffusion coefficient (ADC) map MR images using U-Nets. Med Phys. 2019; 46 (7): $3078-3090$. Available from: doi: 10.1002/mp.13550 .

121. Liu, Y.; Sung, K.; Yang, G.; Afshari Mirak, S.; Hosseiny, M.; Azadikhah, A. et al. Automatic Prostate Zonal Segmentation Using Fully Convolutional Network With Feature Pyramid Attention. IEEE access. 2019; 7 163626-163632. Available from: doi: 10.1109/ACCESS.2019.2952534 .

122. Nie, D.; Wang, L.; Gao, Y.; Lian, J.; Shen, D. STRAINet: Spatially Varying sTochastic Residual AdversarIal Networks for MRI Pelvic Organ Segmentation. IEEE Trans Neural Netw Learn Syst. 2019; 30 (5): 1552-1564. Available from: doi: 10.1109/TNNLS.2018.2870182.

123. Taghanaki, S.A.; Zheng, Y.; Kevin Zhou, S.; Georgescu, B.; Sharma, P.; Xu, D. et al. Combo loss: Handling input and output imbalance in multi-organ segmentation. Comput Med Imaging Graph. 2019; 75 24-33. Available from: doi: 10.1016/j.compmedimag.2019.04.005 .

124. Elguindi, S.; Zelefsky, M.J.; Jiang, J.; Veeraraghavan, H.; Deasy, J.O.; Hunt, M.A. et al. Deep learning-based auto-segmentation of targets and organs-at-risk for magnetic resonance imaging only planning of prostate radiotherapy. Phys Imaging Radiat Oncol. 2019; 12 80-86. Available from: doi: 10.1016/j.phro.2019.11.006 .

125. Tan, L.; Liang, A.; Li, L.; Liu, W.; Kang, H.; Chen, C. Automatic prostate segmentation based on fusion between deep network and variational methods. J Xray Sci Technol. 2019; 27 (5): 821-837. Available from: doi: 10.3233/XST-190524 .

126. Yan, K.; Wang, X.; Kim, J.; Khadra, M.; Fulham, M.; Feng, D. A propagation-DNN: Deep combination learning of multi-level features for MR prostate segmentation. Comput Methods Programs Biomed. 2019; 170 11-21. Available from: doi: 10.1016/j.cmpb.2018.12.031.

127. Zhu, Y.; Wei, R.; Gao, G.; Ding, L.; Zhang, X.; Wang, X. et al. Fully automatic segmentation on prostate MR images based on cascaded fully convolution network. J Magn Reson Imaging. 2019; 49 (4): 1149-1156. Available from: doi: 10.1002/jmri.26337 .

128. Alkadi, R.; Alkadi, R.; Taher, F.; Taher, F.; El-baz, A.; El-baz, A. et al. A Deep Learning-Based Approach for the Detection and Localization of Prostate Cancer in T2 Magnetic Resonance Images. J Digit Imaging. 2019; 32 (5): 793-807. Available from: doi: 10.1007/s10278-018-0160-1 .

129. Ghavami, N.; Hu, Y.; Gibson, E.; Bonmati, E.; Emberton, M.; Moore, C.M. et al. Automatic segmentation of prostate MRI using convolutional neural networks: Investigating the impact of network architecture on the accuracy of volume measurement and MRI-ultrasound registration. Med Image Anal. 2019; 58 101558. Available from: doi: 10.1016/j.media.2019.101558 .

130. Zhang, Y.; Wu, J.; Chen, W.; Chen, Y.; Tang, X. Prostate Segmentation Using Z-Net. In 2019 IEEE 16th International Symposium on Biomedical Imaging (ISBI 2019); 2019; 11-14.

131. Feng, Z.; Nie, D.; Wang, L.; Shen, D. Semi-supervised learning for pelvic MR image segmentation based on multi-task residual fully convolutional networks. Proc IEEE Int Symp Biomed Imaging. 2018; 885-888. Available from: doi: 10.1109/ISBI.2018.8363713

132. Han, C.; Zhang, J.; Hataya, R.; Nagano, Y.; Nakayama, H.; Rundo, L. Prostate zonal segmentation using deep learning. 2018.

133. Brosch, T.; Peters, J.; Groth, A.; Stehle, T., Weese, J. Deep Learning-Based Boundary Detection for Model-Based Segmentation with Application to MR Prostate Segmentation. In International Conference on Medical Image Computing and Computer-Assisted Intervention. Springer, Cham.. Cham: Springer International Publishing; 2018; 515-522.

134. Kang, J.; Samarasinghe, G.; Senanayake, U.; Conjeti, S.; Sowmya, A. Deep Learning for Volumetric Segmentation in SpatioTemporal Data: Application to Segmentation of Prostate in DCE-MRI. In 2019 IEEE 16th International Symposium on Biomedical Imaging (ISBI 2019); 2019; 61-65.

135. Drozdzal, M.; Chartrand, G.; Vorontsov, E.; Shakeri, M.; Di Jorio, L.; Tang, A. et al. Learning normalized inputs for iterative estimation in medical image segmentation. Med Image Anal. 2018; 44, 1-13. Available from: doi: 10.1016/j.media.2017.11.005 .

136. To, M.N.N.; Vu, D.Q.; Turkbey, B.; Choyke, P.L.; Kwak, J.T. Deep dense multi-path neural network for prostate segmentation in magnetic resonance imaging. Int J Comput Assist Radiol Surg. 2018; 13 (11): 1687-1696. Available from: doi: 10.1007/s11548018-1841-4 .

137. Karimi, D.; Samei, G.; Kesch, C.; Nir, G.; Salcudean, S.E. Prostate segmentation in MRI using a convolutional neural network architecture and training strategy based on statistical shape models. Int J Comput Assist Radiol Surg. 2018; 13 (8): $1211-1219$. Available from: doi: 10.1007/s11548-018-1785-8 .

138. Zhu, Q.; Du, B.; Turkbey, B.; Choyke, P.L.; Yan, P. Deeply-supervised CNN for prostate segmentation. 2017 international joint conference on neural networks (IJCNN). IEEE; 2017; 178-184.

139. Zhu, Y.; Wang, L.; Liu, M.; Qian, C.; Yousuf, A.; Oto, A. et al. MRI-based prostate cancer detection with high-level representation and hierarchical classification. Med Phys. 2017; 44 (3): 1028-1039. Available from: doi: 10.1002/mp.12116 .

140. Cheng, R.; Roth, H.R.; Lay, N.; Lu, L.; Turkbey, B.; Gandler, W. et al. Automatic magnetic resonance prostate segmentation by deep learning with holistically nested networks. J.Med.Imag. 2017; 4 (4): 041302. Available from: doi: 10.1117/1.JMI.4.4.041302 .

141. Clark, T.; Zhang, J.; Baig, S.; Wong, A.; Haider, M.A.; Khalvati, F. Fully automated segmentation of prostate whole gland and transition zone in diffusion-weighted MRI using convolutional neural networks. J.Med.Imag. 2017; 4 (4): 041307. Available from: doi: 10.1117/1.JMI.4.4.041307.

142. Yu, L.; Yang, X.; Chen, H.; Qin, J.; Heng, P. Volumetric ConvNets with Mixed Residual Connections for Automated Prostate Segmentation from 3D MR Images. In Proceedings of the AAAI Conference on Artificial Intelligence. 2017; 31 (1). 
143. Guo, Y.; Gao, Y.; Shen, D. Deformable MR Prostate Segmentation via Deep Feature Learning and Sparse Patch Matching. IEEE Trans Med Imaging. 2016; 35 (4): 1077-1089. Available from: doi: 10.1109/TMI.2015.2508280 .

144. Liao, S.; Gao, Y.; Oto, A.; Shen, D. Representation Learning: A Unified Deep Learning Framework for Automatic Prostate MR Segmentation. In International Conference on Medical image computing and computer-assisted intervention. Berlin, Heidelberg: Springer Berlin Heidelberg; 2013; 254-261.

145. Men, K.; Boimel, P.; Janopaul-Naylor, J.; Zhong, H.; Huang, M.; Geng, H. et al. Cascaded atrous convolution and spatial pyramid pooling for more accurate tumor target segmentation for rectal cancer radiotherapy. Phys.Med.Biol. 2018; 63 (18): 185016. Available from: doi: 10.1088/1361-6560/aada6c .

146. Men, K.; Dai, J.; Li, Y. Automatic segmentation of the clinical target volume and organs at risk in the planning CT for rectal cancer using deep dilated convolutional neural networks. Med phys. 2017; 44 (12): 6377-6389. Available from: doi: 10.1002/mp.12602 .

147. Zhao, X.; Xie, P.; Wang, M.; Li, W.; Pickhardt, P.J.; Xia, W. et al. Deep learning-based fully automated detection and segmentation of lymph nodes on multiparametric-mri for rectal cancer: A multicentre study. EBioMedicine. 2020; 56 102780. Available from: doi: 10.1016/j.ebiom.2020.102780 .

148. Wang, M.; Xie, P.; Ran, Z.; Jian, J.; Zhang, R.; Xia, W. et al. Full convolutional network based multiple side-output fusion architecture for the segmentation of rectal tumors in magnetic resonance images: A multi-vendor study. Med Phys. 2019; 46 (6): 26592668. Available from: doi: 10.1002/mp.13541 .

149. Wang, J.; Lu, J.; Qin, G.; Shen, L.; Sun, Y.; Ying, H. et al. Technical Note: A deep learning-based autosegmentation of rectal tumors in MR images. Med Phys. 2018; 45 (6).

150. Huang, Y.; Dou, Q.; Wang, Z.; Liu, L.; Wang, L.; Chen, H. et al. HL-FCN: Hybrid loss guided FCN for colorectal cancer segmentation. In 2018 IEEE 15th International Symposium on Biomedical Imaging (ISBI 2018); 2018; 195-198.

151. Trebeschi, S.; van Griethuysen, J.J.M.; Lambregts, D.M.J.; Lahaye, M.J.; Parmer, C.; Bakers, F.C.H. et al. Deep Learning for FullyAutomated Localization and Segmentation of Rectal Cancer on Multiparametric MR. Sci Rep. 2017; 7 (1): 5301-9. Available from: doi: 10.1038/s41598-017-05728-9 .

152. McVeigh, P.Z.; McVeigh, P.Z.; Syed, A.M.; Syed, A.M.; Milosevic, M.; Milosevic, M. et al. Diffusion-weighted MRI in cervical cancer. Eur Radiol. 2008; 18 (5): 1058-1064. Available from: doi: 10.1007/s00330-007-0843-3 .

153. Liu, Z.; Liu, X.; Xiao, B.; Wang, S.; Miao, Z.; Sun, Y. et al. Segmentation of organs-at-risk in cervical cancer CT images with a convolutional neural network. Physica Medica. 2020; 69 184-191. Available from: doi: 10.1016/j.ejmp.2019.12.008 .

154. Niaf, E.; Rouvière, O.; Mège-Lechevallier, F.; Bratan, F.; Lartizien, C. Computer-aided diagnosis of prostate cancer in the peripheral zone using multiparametric MRI. Phys.Med.Biol. 2012; 57 (12): 3833-3851. Available from: doi: 10.1088/00319155/57/12/3833.

155. Toth, R.; Ribault, J.; Gentile, J.; Sperling, D.; Madabhushi, A. Simultaneous segmentation of prostatic zones using Active Appearance Models with multiple coupled levelsets. Comput Vis Image Underst. 2013; 117 (9): 1051-1060. Available from: doi: 10.1016/j.cviu.2012.11.013.

156. Qiu, W.; Yuan, J.; Ukwatta, E.; Sun, Y.; Rajchl, M.; Fenster, A. Dual optimization based prostate zonal segmentation in 3D MR images. Med Image Anal. 2014; 18 (4): 660-673. Available from: doi: 10.1016/j.media.2014.02.009 .

157. Makni, N.; Iancu, A.; Colot, O.; Puech, P.; Mordon, S.; Betrouni, N. Zonal segmentation of prostate using multispectral magnetic resonance images: Zonal segmentation of prostate using multispectral MR images. Med phys. 2011; 38 (11): 6093-6105. Available from: doi: 10.1118/1.3651610 .

158. Litjens, G.; Toth, R.; van de Ven, W.; Hoeks, C.; Kerkstra, S., van Ginneken, B. et al. Evaluation of prostate segmentation algorithms for MRI: The PROMISE12 challenge. Med Image Anal. 2014; 18 (2): 359-373. Available from: doi: 10.1016/j.media.2013.12.002 .

159. Bloch, N.; Madabhushi, A.; Huisman, H.; Freymann, J.; Kirby, J.; Grauer, M. et al. NCI-ISBI 2013 Challenge: Automated Segmentation of Prostate Structures. 2013.

160. Litjens, G.; Debats, O.; Barentsz, J.; Karssemeijer, N.; Huisman, H. ProstateX Challenge database. 2017.

161. Yu, L.; Cheng, J.; Dou, Q.; Yang, X.; Chen, H.; Qin, J. et al. Automatic 3D Cardiovascular MR Segmentation with DenselyConnected Volumetric ConvNets. In International conference on medical image computing and computer-assisted intervention. Springer, Cham.; 2017; 287-295.

162. Milletari, F.; Navab, N.; Ahmadi, S. V-Net: Fully Convolutional Neural Networks for Volumetric Medical Image Segmentation. In 2016 fourth international conference on $3 D$ vision (3DV); 2016; 565-571.

163. Lemaître, G.; Martí, R.; Freixenet, J.; Vilanova, J.C.; Walker, P.M.; Meriaudeau, F. Computer-Aided Detection and diagnosis for prostate cancer based on mono and multi-parametric MRI: A review. Comput Biol Med. 2015; 60 8-31. Available from: doi: 10.1016/j.compbiomed.2015.02.009.

164. Saidu, C.I.; Csató, L. Medical Image Analysis with Semantic Segmentation and Active Learning. Universitatis Babes-Bolyai Informatica. 2019; 26.

165. The Brigham and Women's Hospital, (BWH). BWH Prostate MR Image Database. 2008.

166. Pekar, V.; McNutt, T.R.; Kaus, M.R. Automated model-based organ delineation for radiotherapy planning in prostatic region. Int J Radiat Oncol Biol Phys. 2004; 60 (3): 973-980. Available from: doi: 10.1016/j.ijrobp.2004.06.004 .

167. Pasquier, D.; Lacornerie, T.; Vermandel, M.; Rousseau, J.; Lartigau, E.; Betrouni, N. Automatic Segmentation of Pelvic Structures From Magnetic Resonance Images for Prostate Cancer Radiotherapy. Int J Radiat Oncol Biol Phys. 2007; 68 (2): 592-600. Available from: doi: 10.1016/j.ijrobp.2007.02.005 . 
168. Dong, X.; Lei, Y.; Tian, S.; Wang, T.; Patel, P.; Curran, W.J. et al. Synthetic MRI-aided multi-organ segmentation on male pelvic CT using cycle consistent deep attention network. Radiother Oncol. 2019; 141: 192-199. Available from: doi: 10.1016/j.radonc.2019.09.028 .

169. Kaur, H.; Choi, H.; You, Y.N.; Rauch, G.M.; Jensen, C.T.; Hou, P. et al. MR imaging for preoperative evaluation of primary rectal cancer: practical considerations. Radiographics; Radiographics. 2012; 32 (2): 389-409. Available from: doi: 10.1148/rg.322115122 .

170. Hernando-Requejo, O.; López, M.; Cubillo, A.; Rodriguez, A.; Ciervide, R.; Valero, J. et al. Complete pathological responses in locally advanced rectal cancer after preoperative IMRT and integrated-boost chemoradiation. Strahlenther Onkol. 2014; 190 (6): 515-520. Available from: doi: 10.1007/s00066-014-0650-0 .

171. Schipaanboord, B.; Boukerroui, D.; Peressutti, D.; van Soest, J.; Lustberg, T.; Dekker, A. et al. An Evaluation of Atlas Selection Methods for Atlas-Based Automatic Segmentation in Radiotherapy Treatment Planning. IEEE Trans Med Imaging. 2019; 38 (11): 2654-2664. Available from: doi: 10.1109/TMI.2019.2907072 .

172. Fritscher, K.D.; Peroni, M.; Zaffino, P.; Spadea, M.F.; Schubert, R.; Sharp, G. Automatic segmentation of head and neck CT images for radiotherapy treatment planning using multiple atlases, statistical appearance models, and geodesic active contours. Med Phys. 2014; 41 (5): 051910-n/a. Available from: doi: 10.1118/1.4871623 .

173. Losnegård, A.; Hysing, L.B.; Kvinnsland, Y.; Muren, L.P.; Munthe-Kaas, A.; Hodneland, E. et al. Semi-Automatic Segmentaiton of the Large Intestine for Radiotherapy Planning Using the Fast-Marching Method. Radiother Oncol. 2012; 92 S75. Available from: doi: 10.1016/S0167-8140(12)72788-3 .

174. Haas, B.; Coradi, T.; Scholz, M.; Kunz, P.; Huber, M.; Oppitz, U. et al. Automatic segmentation of thoracic and pelvic CT images for radiotherapy planning using implicit anatomic knowledge and organ-specific segmentation strategies. Phys Med Biol. 2008; 53 (6): 1751-1771. Available from: doi: 10.1088/0031-9155/53/6/017 .

175. Gambacorta, M.; Valentini, C.; Dinapoli, N.; Mattiucci, G.C.; Pasini, D.; Barba, M.C. et al. Atlas-based Auto-segmentation Clinical Validation of Pelvic Volumes and Normal Tissue in Rectal Tumors. Int J Radiat Oncol Biol Phys. 2012; 84 (3): S347-S348. Available from: doi: 10.1016/j.ijrobp.2012.07.916.

176. Malayeri, A.A.; El Khouli, R.H.; Zaheer, A.; Jacobs, M.A.; Corona-Villalobos, C.; Kamel, IR. et al. Principles and applications of diffusion-weighted imaging in cancer detection, staging, and treatment follow-up. Radiographics. 2011; 31 (6): 1773-1791. Available from: doi: 10.1148/rg.316115515 .

177. Ma, D.; Gulani, V.; Seiberlich, N.; Liu, K.; Sunshine, J.L.; Duerk, J.L. et al. Magnetic resonance fingerprinting. Nature. 2013; 495 (7440): 187-192.

178. O'Connor, J.P.; Aboagye, E.O.; Adams, J.E.; Aerts, H.J.; Barrington, S.F.; Beer, A.J. et al. Imaging biomarker roadmap for cancer studies. Nature reviews Clinical oncology; 2016; 14 (3), 169.

179. Oakden-Rayner, L. Exploring Large-scale Public Medical Image Datasets. Acad Radiol. 2020; 27 (1): 106-112. Available from: doi: 10.1016/j.acra.2019.10.006.

180. Cuocolo, R.; Stanzione, A.; Castaldo, A.; De Lucia, D.R.; Imbriaco, M. Quality control and whole-gland, zonal and lesion annotations for the PROSTATEx challenge public dataset. Eur J Radiol. 2021; 138 109647. Available from: doi: 10.1016/j.ejrad.2021.109647. 